\title{
Presentation in Self-Posted Facial Images Can Expose Sexual Orientation: Implications for Research and Privacy
}

\author{
Dawei Wang ${ }^{1^{*}}$ \\ ${ }^{1}$ Kellogg School of Management, Northwestern University; \\ ${ }^{*}$ Corresponding Author. Email: david.wang@kellogg.northwestern.edu
}

Forthcoming at Journal of Personality and Social Psychology, Attitudes and Social Cognition Section

Please note that this preprint is not the final proof that will appear in the APA journal. If you are interested in citing this article, please contact the author. 


\begin{abstract}
Recent research has found that facial recognition algorithms can accurately classify people's sexual orientations using naturalistic facial images, highlighting a severe risk to privacy. This article tests whether people of different sexual orientations presented themselves distinctively in photographs, and whether these distinctions revealed their sexual orientation. I found significant differences in self-presentation. For example, gay individuals were on average more likely to wear glasses compared to heterosexual individuals in images uploaded to the dating website. Gay men also uploaded brighter images compared to heterosexual men. To further test how some of these differences drove the classification of sexual orientation, I employed image augmentation or modification techniques. To evaluate whether the image background contributed to classifications, I progressively masked images until only a thin border of image background remained in each facial image. I found that even these pixels classified sexual orientations at rates significantly higher than random chance. I also blurred images, and found that merely three numbers representing the brightness of each color channel classified sexual orientations. These findings contribute to psychological research on sexual orientation by highlighting how people chose to present themselves differently on the dating website according to their sexual orientations, and how these distinctions were used by the algorithm to classify sexual orientations. The findings also expose a privacy risk as they suggest that do-it-yourself data-protection strategies, such as masking and blurring, cannot effectively prevent leakage of sexual orientation information. As consumers are not equipped to protect
\end{abstract}


themselves, the burden of privacy protection should be shifted to companies and governments.

Keywords: facial recognition, sexual orientation, privacy, impression management, self-presentation, facial perception 


\section{Presentation in Self-Posted Facial Images Can Expose Sexual Orientation: Implications for Research and Privacy}

Several recent studies have found that sensitive personal attributes are becoming increasingly easy to detect using facial images. Advanced facial recognition algorithms can now accurately classify sensitive traits, such as sexual orientation (Wang \& Kosinski, 2018), personality (Kachur et al., 2020; Wolffhechel et al., 2014), political orientation (Kosinski, 2021), and unlawful behaviors (Wu \& Zhang, 2017). For example, Wang and Kosinski (2018) found that an off-the-shelf facial recognition algorithm can be easily repurposed into a sexual orientation classifier that can differentiate sexual orientation with a classification rate ${ }^{1}$ of above $80 \%$ for men and $70 \%$ for women from a single naturalistic facial image, considerably more accurate than what can be achieved by human judges. In another study, a similar off-the-shelf algorithm was shown to classify individuals' political orientation in Facebook and dating profile images with a classification rate of over $70 \%$ (Kosinski, 2021).

What is unclear is to what extent the classifications were driven by fixed (i.e., facial morphology), transient (i.e., grooming styles), and non-facial, image features (e.g., background or lighting). Facial recognition research posits that if faces were aligned at the same position in the facial images used to train the algorithm, each pixel in the image would map on to a specific facial feature (Parkhi et al., 2015; Taigman et al., 2015). Similarly, Taigman et al. (2015) referred to their algorithm as "a well localized description of the underlying face" because they found that pixels that activated the

\footnotetext{
${ }^{1}$ Classification rate is expressed as area under the receiver operating characteristic curve (AUC). When presented with stimulus $X$ from category $A$ and stimulus $Y$ from category $B$, the AUC refers to the extent to which the model assigns $Y$ a higher probability of belonging to category $B$ than $X$.
} 
algorithm were in the facial area. Building on these findings, Wu and Zhang (2016, p. 2) claimed that "sophisticated algorithms based on machine learning may discover very delicate and elusive nuances in facial characteristics and structures that correlate to innate personal traits." Stoker et al. (2016, p. 8) even described machine learning as an "advanced objective method for the measurement of facial features."

On the other hand, some researchers acknowledged the contribution of both facial morphology and grooming features. For example, Wang and Kosinski (2018) explained that "[f]acial features employed by the classifier included both fixed (e.g., nose shape) and transient facial features (e.g., grooming style)" (p. 246), although they traced some of these differences to biological predispositions, citing the prenatal hormonal hypothesis (e.g., "According to the PHT [prenatal hormonal theory], samegender sexual orientation stems from the underexposure of male fetuses or overexposure of female fetuses to androgens that are responsible for sexual differentiation"; p. 247). Kachur et al. (2020) claimed that "machine learning... could reveal multidimensional personality profiles based on static morphological facial features" but the researchers were "still unable to claim that morphological features of the face explain all the personality-related image variance captured by the ANNs" (p. 6). Agüera y Arcas et al. (2018) surveyed 8,000 Americans of different sexual orientations and asked them to fill out an array of "yes/no" questions about their self-presentational style. The results showed that gay subjects were more likely to report wearing glasses and less likely to report having face tan and working outdoors.

These findings are consistent with the impression management literature, which posits that people intentionally or unintentionally shape how they are seen by others 
(Goffman, 1959; Leary \& Allen, 2011; Leary \& Kowalski, 1990; Schau \& Gilly, 2003;

Schlenker, 2012). For example, people choose to present themselves differently by constructing distinctive text-based self-descriptions on online dating platforms (Schau \& Gilly, 2003; Tong et al., 2020). Self-presentational studies also found in unfamiliar and different-sex social interactions, people felt more nervous, thought about others' impressions more, and wanted to make better impressions than they did in familiar and/or same-sex interactions (Leary et al., 1994). When sexual orientation comes into play, gay and heterosexual men have distinctive aesthetic appeals (Rudd, 1996). Gay men on average preferred innovative or trendy apparel, whereas heterosexual men preferred casual or laid-back styles.

The idea that people choose different photographs depending on the context is not new in facial perception literature. Todorov and Porter (2014) explained that "[w]ebsite users did not randomly select which images of themselves to post on these sites. Hence, it is possible that the presumed accuracy reflects biases in the selection of the images rather than honest or inherent signals of sexual orientation in the face" ( $p$. 1415). The authors asked participants to select images of different facial expressions and found that in scenarios such as dating, participants chose photographs that portrayed a trustworthy-looking face compared to a mean-looking face of the same person. These findings were confirmed by White et al. (2017), who asked participants to upload 12 photographs of themselves to a professional and a dating website. Participants systematically uploaded more attractive-looking photographs to the dating website and more competent-looking photographs to the professional website. Hancock and Toma (2009) found that in online dating photographs, people engaged in self- 
enhancement tactics to make themselves appear more attractive. These tactics varied by gender; women posted photographs of themselves taken when they were younger, and were more likely to re-touch their photographs compared to men.

Understanding how presentation in self-posted facial images influences classification of sexual orientation is critical to the ongoing discussion about privacy (Matz et al., 2020). If trait classifications were mostly driven by morphological differences, as implied in existing facial recognition research, privacy loss would be preventable using existing de-identification technologies. For example, consumers can protect themselves using do-it-yourself data protection software that masks, blurs, or pixelates the facial regions of these images, or by wearing face masks (Li et al., 2017; Matzner et al., 2016; Shan et al., 2020; Zhang et al., 2014). However, if classifications were also driven by self-presentation, the danger of privacy loss might be greater than previously believed. There are more dimensions on which self-presentation may vary. Pinpointing the exact features in the facial image where private information is retrieved would be difficult. In this case, the burden of privacy protection must be shifted to governments and companies because the alternative to privacy protection would be to ask consumers to refrain from self-expressions (i.e., not post images online), a tradeoff that many might not want to make.

To understand how self-presentation influences the ability to extract sexual orientation information from self-posted photographs, I collected a dataset consisting of 15,286 gay and heterosexual participants from an online dating website. I first obtained 12 self-presentational facial attributes from their facial images, tested whether there were significant differences according to sexual orientation (Study 1a), and examined 
the degree these differences contributed to classification of sexual orientation (Study 1b). Then, I replicated the sexual orientation classification algorithm. I tested the contribution of image background on the classification of sexual orientation (Study 1c). I masked the facial portion in each facial image so that only background information remained. If masked images, in which only the image background was retained, can classify sexual orientation, it means that the image background (a self-presentational feature) contributed to sexual orientation classification.

Next, previous research suggests that gay men appeared to have brighter skin tone compared to heterosexual men. I tested whether skin tone was related to the overall brightness of the face and/or the background (Study 2a). If skin tone was related to overall brightness (both face and background), it is likely that gay vs. heterosexual people presented themselves in images with varying levels of brightness or illumination. Finally, I tested the contribution of overall image brightness on sexual orientation classification (Study 2b). I blurred each facial image so that only three numbers representing the brightness of each color channel remained. If a completely blurred out image can classify sexual orientation, then people's choices of image brightness, or the illumination of their ambient environments (indoor vs. outdoors), contributed to sexual orientation classification.

\section{Study 1: Differences across Sexual Orientations in Self-Presentational Attributes Extracted Using the Face++ API}

Agüera y Arcas et al. (2018) found that reported self-presentational styles varied by sexual orientation. Some self-presentational differences were also reported by Wang and Kosinski (2018), such as the likelihood of wearing glasses in facial images. Study 
1a aimed to extend their findings and test the extent these differences are observed in self-posted facial images on a dating website.

\section{Methods}

Preprocessing Facial Images. All data collection was conducted after the study was approved by the institutional review board (IRB) of my university. Following the exact procedures described in Wang and Kosinski (2018), I collected facial images of public profiles from a U.S. dating website. I aimed to collect a larger sample than the previous study to achieve higher generalizability. I gathered a total of 76,181 profiles (412,446 images), of which 39,386 were women (224,855 facial images) and 36,795 were men (187,591 facial images), aged 18 to 40 .

Next, I cleaned and preprocessed all facial images with the help of the Face++ API, which is a facial recognition software widely used in facial research (Kosinski, 2021; Wang et al., 2019; Wang \& Kosinski, 2018), verified to be accurate at extracting facial information from images (Jaeger et al., 2020). Four sets of information were extracted: the number of faces in each facial image, facial landmarks, facial attributes, and facial bounding boxes. Like Wang and Kosinski (2018), I dropped images that did not contain human faces, contained more than one face, contained partially hidden faces (i.e., if any occlusions were detected by the Face++ API), or had small-resolution faces (i.e., the width of the bounding box was less than 40 pixels). Like the previous study, I also removed images in which faces were significantly turned away from the camera (i.e., head pose yaw angle greater than 15 degrees and pitch angle greater than 10 degrees). All these steps were taken to ensure that every face in the dataset would 
align in the same position and angle, allowing the facial recognition algorithm to accurately recognize the face.

Following Wang and Kosinski (2018), I only included individuals who are Caucasians within the age of 18 to 40 . However, instead of verifying the demographics manually, I relied on the gender and age detector of Face++ API, as well as a pretrained ethnicity detector in the DeepFace algorithm (Serengil \& Ozpinar, 2020). I removed individuals whose mode-apparent gender from all facial images did not fit their reported gender category. This is because there were some individuals who selfidentified as the opposite gender. I removed individuals whose mode-apparent ethnicity was not Caucasian to retain only Caucasians in the sample like Wang and Kosinski (2018). I also excluded individuals whose average-apparent age detected from their photographs was not within the 18 to 40 age range. To ensure that the resulting gender and ethnicity in the dataset were accurate, I randomly drew 100 individuals from each gender-sexual-orientation category (a total of 400 images). All genders were perfectly classified. Only two people's ethnicities 2 out of the 400 might be incorrectly classified (99.5\% accuracy).

Machine learning research recommends balancing the training data to prevent bias towards the majority group (Susan \& Kumar, 2021). Thus, I matched the sample size, age, and number of images of the sub-samples using an automated process. The process, conducted separately for each gender, paired every person from a sexual

\footnotetext{
${ }^{2}$ One heterosexual man who appears to be multiracial and one heterosexual man who appears to be Latino were considered as misclassified. Note that this accuracy rate is high because strict inclusion criteria were used, i.e., included only if the mode ethnicity classification from all facial images of each person was Caucasian. Note also that Latinos were not considered as Caucasian by the DeepFace algorithm and by my manual accuracy check even when some might have identified themselves as Caucasian. Latinos were not included to avoid ambiguity.
} 
orientation group with a person from the other group by age and number of images. The resulting number of gay versus heterosexual people, as well as the number of their facial images, were completely balanced. The final sample contained 10,162 facial images of 5,124 men (50\% gay and 50\% heterosexual) and 21,600 facial images of 10,340 women ( $50 \%$ gay and $50 \%$ heterosexual). A breakdown of the final sample and age distributions is reported in Table 1.

Finally, I cropped and aligned all facial images using the bounding box provided by Face++ API. All resulting facial images in the dataset contained facial positions that matched exactly those reported in Figure 4 of Wang \& Kosinski (2018). All images were resized to 224 by 224 pixels as required by the facial recognition algorithm used to classify sexual orientation.

\section{Table 1.}

Sample Breakdown by Users and Number of Facial Images for Studies 1a, 1b, 1c, 2a and $2 b$

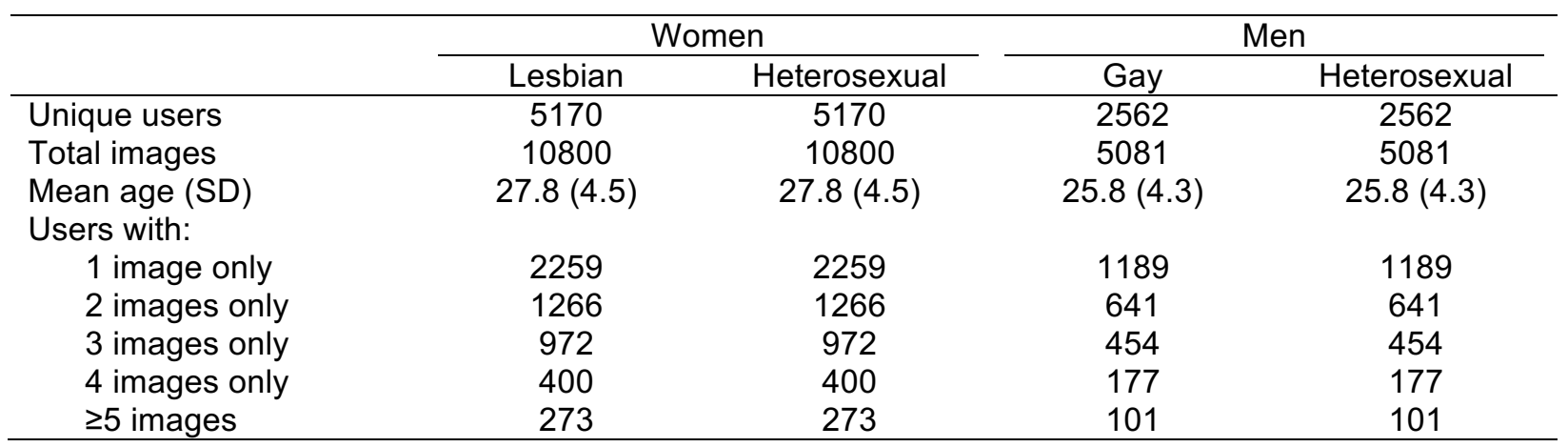

Self-Presentational Attributes. A total of 12 self-presentational facial attributes was extracted from Face++ API to test whether there was a significant difference in these features according to sexual orientation (Study 1a), and whether this difference contributed to classification of sexual orientation (Study 1b). All attributes except head 
pose angles were measured in probabilities, i.e., how likely the face found in an image displayed a certain attribute. All attributes were standardized to a range of zero and one for easier comparisons. As participants varied on the number of facial images, a different number of sets of attributes was produced for each participant. The withinperson reliability of these attributes was moderate (see Table S1 of Supplementary Materials), so within-person attribute sets were averaged so that each participant only had one set of facial attributes.

All six facial expression scores, such as happy and neutral, were included. These scores measured the probability that the face in each image displayed a certain facial expression. Research has shown that head pose angles are related to different facial perceptions and emotional expressivity (Barrett et al., 2019; Nicholls et al., 2002; Witkower \& Tracy, 2019). Thus, head pose angles consisting of the absolute roll, yaw, and pitch angles were included. Research found that gay individuals reported being more likely to wear glasses than heterosexual individuals (Agüera y Arcas et al., 2018; Wang \& Kosinski, 2018). Thus, the probability of wearing glasses was included. Finally, eye status, the probability of eyes being open, and the probability of a smile being present in the image, were included.

Transparency and Openness. Data include sensitive personal information, thus would not be disclosed. The code is available at https://osf.io/q39py/. All materials are included in the main text and supplementary materials. There are no additional materials to disclose. The design and analysis of this study were not pre-registered.

\section{Results}


Table 2 reports the mean, confidence intervals, and statistical tests of facial attributes. Figure 1 shows the differences in means ranked from positive to negative. All results were reported separately for women and men.

Table 2.

Comparison of the Means of Self-Presentational Facial Attributes by Sexual Orientation and Gender for Study $1 a$

\begin{tabular}{|c|c|c|c|c|c|c|c|c|c|c|c|}
\hline & \multicolumn{11}{|c|}{ Women $(\mathrm{N}=10,340)$} \\
\hline & \multicolumn{3}{|c|}{ Heterosexual } & \multicolumn{3}{|c|}{ Lesbian } & \multicolumn{5}{|c|}{ Significance Test } \\
\hline & \multirow[t]{2}{*}{ Mean } & \multicolumn{2}{|c|}{$95 \% \mathrm{Cl}$} & \multirow[t]{2}{*}{ Mean } & \multicolumn{2}{|c|}{$95 \% \mathrm{Cl}$} & $t$ & \multicolumn{2}{|c|}{$95 \% \mathrm{Cl}$} & \multirow[t]{2}{*}{$p$} & \multirow[t]{2}{*}{$d$} \\
\hline Fac. exp.: & & & & & & & & & & & \\
\hline Neutral & 0.254 & {$[0.24$} & $0.26]$ & 0.325 & {$[0.32$} & $0.34]$ & 10.408 & {$[0.06$} & $0.08]$ & $<.001$ & 0.205 \\
\hline Happiness & 0.622 & {$[0.61$,} & $0.63]$ & 0.522 & {$[0.51$} & $0.53]$ & -12.804 & {$[-0.12$} & $-0.08]$ & $<.001$ & 0.252 \\
\hline Anger & 0.015 & {$[0.01$,} & $0.02]$ & 0.016 & {$[0.01$,} & $0.02]$ & 1.263 & {$[-0.00$} & $0.00]$ & .206 & 0.025 \\
\hline Disgust & 0.016 & {$[0.01$,} & $0.02]$ & 0.020 & {$[0.02$,} & $0.02]$ & 1.938 & {$[-0.00$} & $0.01]$ & .053 & 0.038 \\
\hline Surprise & 0.049 & {$[0.05$,} & $0.05]$ & 0.060 & {$[0.06$} & $0.06]$ & 3.457 & {$[0.00$} & $0.02]$ & $<.001$ & 0.068 \\
\hline Sadness & 0.031 & {$[0.03$,} & $0.03]$ & 0.039 & {$[0.04$} & $0.04]$ & 3.251 & {$[0.00$} & $0.01]$ & $<.001$ & 0.064 \\
\hline \multicolumn{12}{|l|}{ Head pose: } \\
\hline Roll (abs) & 0.031 & {$[0.03$} & $0.03]$ & 0.030 & {$[0.03$} & $0.03]$ & -2.300 & {$[-0.00$} & $-0.00]$ & .021 & 0.045 \\
\hline Yaw (abs) & 0.458 & {$[0.45$} & $0.46]$ & 0.447 & {$[0.44$} & $0.45]$ & -2.400 & {$[-0.02$} & $-0.00]$ & .016 & 0.047 \\
\hline Pitch (abs) & 0.551 & {$[0.54$} & $0.56]$ & 0.518 & {$[0.51$} & $0.52]$ & -7.143 & {$[-0.04$} & $-0.02]$ & $<.001$ & 0.140 \\
\hline Smiling & 0.680 & {$[0.67$} & $0.69]$ & 0.576 & {$[0.57$} & $0.59]$ & -13.441 & {$[-0.12$} & $-0.09]$ & $<.001$ & 0.264 \\
\hline Eyes & 0.117 & {$[0.11$} & $0.12]$ & 0.158 & {$[0.15$} & $0.17]$ & 7.296 & {$[0.03$} & $0.05]$ & $<.001$ & 0.144 \\
\hline \multirow[t]{4}{*}{ Glasses } & 0.183 & {$[0.17$,} & $0.19]$ & 0.264 & {$[0.25$,} & $0.27]$ & 11.150 & {$[0.07$} & $0.10]$ & $<.001$ & 0.219 \\
\hline & \multicolumn{11}{|c|}{ Men $(\mathrm{N}=5,124)$} \\
\hline & \multicolumn{3}{|c|}{ Heterosexual } & \multicolumn{3}{|c|}{ Gay } & \multicolumn{5}{|c|}{ Significance Test } \\
\hline & Mean & $95^{\circ}$ & & Mean & $95^{\circ}$ & & $t$ & $95^{\circ}$ & & $p$ & $d$ \\
\hline \multicolumn{12}{|l|}{ Fac. exp.: } \\
\hline Neutral & 0.394 & {$[0.38$} & $0.41]$ & 0.441 & {$[0.43$} & $0.46]$ & 4.392 & {$[0.03$} & $0.07]$ & $<.001$ & 0.123 \\
\hline Happiness & 0.479 & {$[0.46$} & $0.49]$ & 0.424 & {$[0.41$,} & $0.44]$ & -4.845 & {$[-0.08$,} & $-0.03]$ & $<.001$ & 0.135 \\
\hline Anger & 0.013 & {$[0.01$,} & $0.02]$ & 0.017 & {$[0.01$,} & $0.02]$ & 1.502 & {$[-0.00$} & $0.01]$ & .133 & 0.042 \\
\hline Disgust & 0.030 & {$[0.03$} & $0.03]$ & 0.029 & {$[0.03$} & $0.03]$ & -0.308 & {$[-0.01$,} & $0.00]$ & .758 & 0.009 \\
\hline Surprise & 0.030 & {$[0.03$,} & $0.03]$ & 0.034 & {$[0.03$} & $0.04]$ & 1.455 & {$[-0.00$} & $0.01]$ & 146 & 0.041 \\
\hline Sadness & 0.036 & {$[0.03$} & $0.04]$ & 0.039 & {$[0.03$} & $0.04]$ & 0.640 & {$[-0.00$} & $0.01]$ & .522 & 0.018 \\
\hline \multicolumn{12}{|l|}{ Head pose: } \\
\hline Roll (abs) & 0.040 & {$[0.04$} & $0.04]$ & 0.041 & {$[0.04$} & $0.04]$ & 1.740 & {$[-0.00$} & $0.00]$ & .082 & 0.049 \\
\hline Yaw (abs) & 0.392 & {$[0.38$} & $0.40]$ & 0.408 & {$[0.40$} & $0.42]$ & 2.400 & {$[0.00$} & $0.03]$ & .016 & 0.067 \\
\hline Pitch (abs) & 0.438 & {$[0.43$,} & $0.45]$ & 0.452 & {$[0.44$} & $0.46]$ & 2.023 & {$[0.00$} & $0.03]$ & .043 & 0.057 \\
\hline Smiling & 0.556 & {$[0.54$,} & $0.57]$ & 0.493 & {$[0.48$} & $0.51]$ & -5.584 & {$[-0.08$,} & $-0.04]$ & $<.001$ & 0.156 \\
\hline Eyes & 0.227 & {$[0.21$,} & $0.24]$ & 0.211 & {$[0.20$} & $0.22]$ & -1.655 & {$[-0.04$} & $0.00]$ & .098 & 0.046 \\
\hline Glasses & 0.235 & {$[0.22$} & $0.25]$ & 0.271 & {$[0.26$} & $0.29]$ & 3.341 & {$[0.02$} & $0.06]$ & $<.001$ & 0.093 \\
\hline
\end{tabular}

As indicated in Table 2, out of the 12 attributes tested in this study, 10 were significantly different across sexual orientations for women and six were different for men ( $p$ 's <.05). As shown by Figure 1, a large difference was observed in the likelihood 
of individuals wearing glasses in facial images. Consistent across both women and men, gay people on average were more likely to upload photographs of themselves wearing glasses compared to heterosexual people in the sample. This is in line with the survey findings in Agüera y Arcas et al. (2018) that gay people on average preferred wearing glasses, as well as the aesthetics of wearing glasses, compared to using contact lenses or not wearing glasses. This is also consistent with the average faces reported in Figure 4 of Wang and Kosinski (2018).

\section{Figure 1.}

Comparison of the Difference of the Means of Self-Presentational Facial Attributes by Sexual Orientation and Gender for Study $1 a$ 

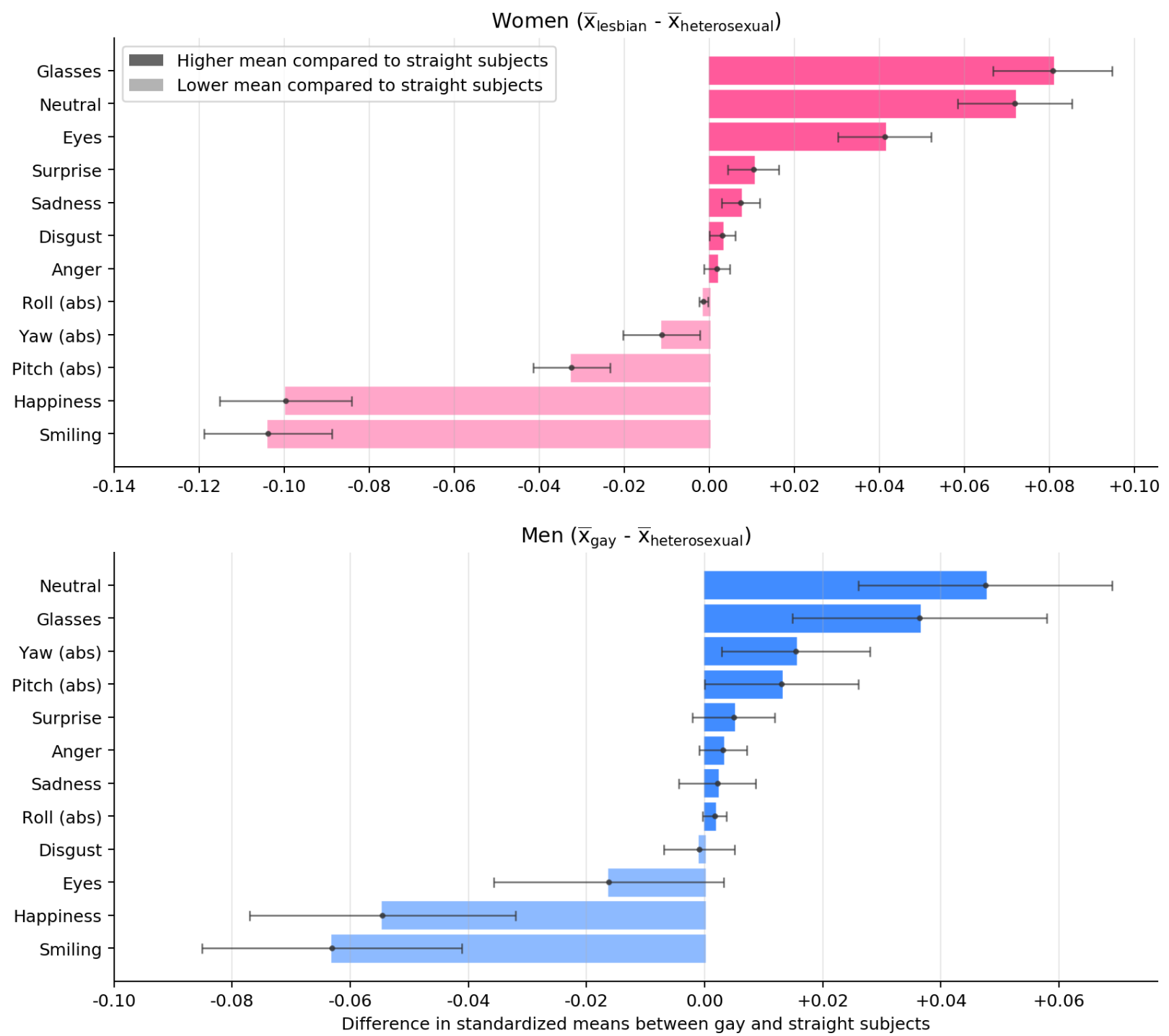

\section{Discussion}

In line with previous findings in the impression management and facial perception literatures, these results demonstrate that there were significant differences in how gay and heterosexual people presented themselves in facial images. My study employed only a few facial attributes from the Face++ API, and found that most attributes differed across sexual orientations. Women on average demonstrated bigger difference across sexual orientations, as seen by the larger effect sizes compared to men. Heterosexual women were more likely than lesbians to display facial actions such as turning the head 
sideways and smiling toward the camera, a facial expression resembling coyness that serves relationship-building functions and helps displayers to connect with observers (Keltner \& Haidt, 1999; Reddy, 2000). This display aligns with previous research in the context of online dating (Todorov \& Porter, 2014). Note that while impression management research posits that self-presentational motifs are typically high in dating contexts (Hancock \& Toma, 2009; Leary et al., 1994; Tong et al., 2020), it is difficult to tell whether the self-presentational styles observed here were intentional or unintentional, and this question is beyond the scope of the study.

\section{Study 1b: Sexual Orientation Classification Using Self-Presentational Attributes Extracted Using the Face++ API}

Study $1 \mathrm{~b}$ aims to examine the extent to which people's sexual orientation could be detected from their self-presentational facial attributes. I trained a logistic regression model using 20 -fold cross-validation. If sexual orientation could be classified at rates above random chance, self-presentation was likely to have contributed to sexual orientation.

\section{Methods}

Self-Presentational Attributes. Study $1 \mathrm{~b}$ employed the same selfpresentational facial attributes extracted using the Face++ API from the dataset of facial images as Study 1a. Like Study 1a, whenever I had multiple images for the same individual, I averaged the attributes across all images.

Sexual Orientation Classifier. I trained a logistic regression model using 20 -fold cross-validation. In each fold, the participants were split into 20 equal parts; 19 parts were used to train the logistic regression while the remaining part was used to classify 
the results. This process ensured that I never used the same data to train and classify the outcome.

\section{Results}

I report the area under the receiver operating characteristic curve (AUC) as a measure of the classification power. AUC is defined as the likelihood that when presented with two images, one from a gay person and one from a heterosexual person, the model would assign the gay person a higher likelihood of being gay than the heterosexual person. I also report the confidence intervals estimated using the Delong method, a general practice in machine learning, deep learning and facial recognition (Delong et al., 1988). I used AUC because it is an evaluation metric widely employed in machine learning research, and was used in previous studies on this topic (Kosinski, 2021; Wang \& Kosinski, 2018). I report other common evaluation metrics in Table 3. All results are reported separately for women and men.

As shown in Figure 2, classification power using all 12 self-presentational facial attributes extracted using Face++ equaled on average $\mathrm{AUC}=.609(95 \% \mathrm{Cl}=$ $[.598, .620])$ for women and $\mathrm{AUC}=.551(95 \% \mathrm{Cl}=[.536, .567])$ for men. The two most predictive attributes were happiness expression and smiling. Happiness expression afforded AUC $=.572(95 \% \mathrm{Cl}=[.561, .583])$ for women and $\mathrm{AUC}=.533(95 \% \mathrm{Cl}=$ $[.517, .549])$ for men. Smiling afforded AUC $=.576(95 \% \mathrm{Cl}=[.565, .587])$ for women and $A \cup C=.544(95 \% \mathrm{Cl}=[.529, .560])$ for men. These results demonstrate that selfpresentational facial attributes extracted using Face++, to some extent, contributed to classification of sexual orientation.

\section{Figure 2.}


Average AUC afforded by Self-Presentational Facial Attributes by Gender for Study $1 b$
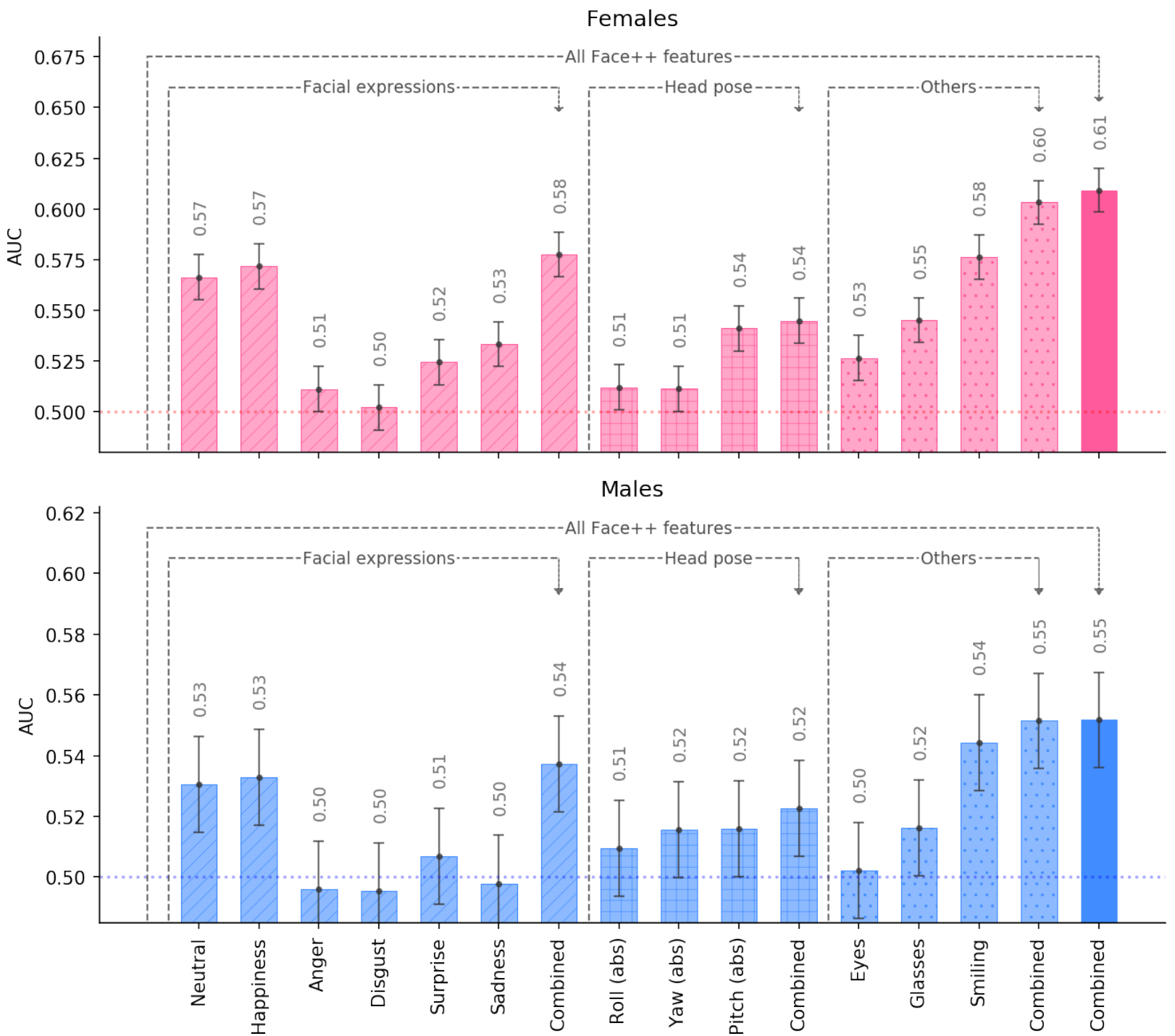

Table 3.

Average Accuracy Afforded by Self-Presentational Facial Attributes by Gender for Study $1 b$

\begin{tabular}{lccccccccc}
\hline & \multicolumn{4}{c}{ Women } & \multicolumn{5}{c}{ Men } \\
\cline { 2 - 4 } & Accuracy & Precision & Recall & F1 & & Accuracy & Precision & Recall & F1 \\
\hline Neutral & $54.93 \%$ & $56.20 \%$ & $44.72 \%$ & $49.81 \%$ & & $52.62 \%$ & $52.73 \%$ & $50.59 \%$ & $51.63 \%$ \\
Happiness & $55.95 \%$ & $56.31 \%$ & $53.08 \%$ & $54.65 \%$ & & $52.97 \%$ & $52.89 \%$ & $54.37 \%$ & $53.62 \%$ \\
Anger & $50.48 \%$ & $52.39 \%$ & $10.62 \%$ & $17.66 \%$ & & $50.35 \%$ & $51.50 \%$ & $12.06 \%$ & $19.54 \%$ \\
Disgust & $50.27 \%$ & $51.31 \%$ & $10.58 \%$ & $17.54 \%$ & & $50.02 \%$ & $50.01 \%$ & $85.21 \%$ & $63.03 \%$ \\
Surprise & $51.39 \%$ & $54.13 \%$ & $18.26 \%$ & $27.31 \%$ & & $50.10 \%$ & $50.38 \%$ & $12.80 \%$ & $20.42 \%$ \\
Sadness & $51.20 \%$ & $54.18 \%$ & $15.53 \%$ & $24.14 \%$ & & $49.75 \%$ & $49.31 \%$ & $18.03 \%$ & $26.41 \%$ \\
$\quad$ Combined & $56.21 \%$ & $56.85 \%$ & $51.55 \%$ & $54.07 \%$ & & $52.48 \%$ & $52.47 \%$ & $52.58 \%$ & $52.52 \%$ \\
\hline Roll (abs) & $50.44 \%$ & $50.37 \%$ & $60.81 \%$ & $55.10 \%$ & $51.19 \%$ & $51.47 \%$ & $41.80 \%$ & $46.13 \%$ \\
\hline
\end{tabular}




\begin{tabular}{lllllllll}
\hline Yaw (abs) & $50.70 \%$ & $50.68 \%$ & $51.90 \%$ & $51.28 \%$ & $51.17 \%$ & $51.25 \%$ & $48.01 \%$ & $49.58 \%$ \\
Pitch (abs) & $53.32 \%$ & $53.45 \%$ & $51.37 \%$ & $52.39 \%$ & $51.07 \%$ & $51.11 \%$ & $49.26 \%$ & $50.17 \%$ \\
Combined & $53.41 \%$ & $53.55 \%$ & $51.55 \%$ & $52.53 \%$ & $52.17 \%$ & $52.24 \%$ & $50.59 \%$ & $51.40 \%$ \\
\hline Eyes & $52.92 \%$ & $56.32 \%$ & $26.02 \%$ & $35.59 \%$ & $51.13 \%$ & $50.84 \%$ & $68.46 \%$ & $58.35 \%$ \\
Glasses & $54.67 \%$ & $57.70 \%$ & $35.01 \%$ & $43.58 \%$ & $51.81 \%$ & $52.76 \%$ & $34.74 \%$ & $41.89 \%$ \\
Smiling & $56.19 \%$ & $57.17 \%$ & $49.38 \%$ & $52.99 \%$ & $53.22 \%$ & $53.17 \%$ & $54.02 \%$ & $53.59 \%$ \\
Combined & $57.85 \%$ & $57.87 \%$ & $57.76 \%$ & $57.81 \%$ & $53.81 \%$ & $53.67 \%$ & $55.66 \%$ & $54.65 \%$ \\
\hline Combined & $58.34 \%$ & $58.54 \%$ & $57.12 \%$ & $57.82 \%$ & $53.47 \%$ & $53.48 \%$ & $53.40 \%$ & $53.44 \%$ \\
\hline
\end{tabular}

\section{Study 1c: Replicating Sexual Orientation Classification Algorithm and Testing How Image Background Contributed to Classifications}

The next step was to find out whether there are other self-presentational factors (i.e., the background) that revealed people's sexual orientation, and directly test their influence on the sexual orientation classification algorithm. I first replicated the algorithm following the exact procedures reported in Wang and Kosinski (2018). Note that both my study and the previous one relied on an off-the-shelf facial recognition algorithm. In other words, no deep neural network training was done. This was intentional because if an algorithm that was totally unrelated to sexual orientation classification could potentially be repurposed to classify sexual orientation, it would suggest a serious risk of privacy loss.

I then tested whether the image background contributed to sexual orientation classification by blocking out the facial portions of the images. Making deliberate adjustments or modifications to an image is called image augmentation in computer vision (Shorten \& Khoshgoftaar, 2019; Zeiler \& Fergus, 2013). In this case, I employed an augmentation technique called masking. If highly masked images, where only background information is retained, could classify sexual orientation at levels significantly higher than chance, it means that people of different sexual orientation are 
presenting themselves by choosing different image backgrounds on online dating websites.

Another goal of this study was to evaluate the limit of privacy protection. Masks are often worn physically in some parts of the world, and used digitally to block people's identities and to prevent the detection of certain private information (Matzner et al., 2016; Zhang et al., 2014). Thus, augmenting the image by masking facial portions aimed to test whether this privacy-protection strategy might prevent the loss of private information. If masks were effective, sexual orientation classification using masked images should drop to chance level. If not, it would imply a serious threat to privacy.

\section{Methods}

Facial Images. Study 1c employed the same dataset of facial images as Study 1a and 1b. However, instead of relying on the self-presentational attributes extracted using the Face++ API, I directly employed the preprocessed facial images.

Sexual Orientation Classifier. I replicated step-by-step procedures employed in Wang and Kosinski (2018) in extracting the deep neural network features, which were later used to identify individuals' sexual orientation. Specifically, I extracted the 4,096 scores for each facial image using the facial recognition algorithm, VGG-Face (Parkhi et al., 2015). Next, the 4,096 scores were reduced to 500 dimensions using the singular value decomposition, a technique like principal component analysis. Finally, the 500 dimension scores were passed through a logistic regression model, with L1-penalty of 1 , to generate the binary sexual orientation classification of gay versus heterosexual. Note that no deep neural network was trained to classify individuals' sexual orientation; 
the network was merely used to convert images into 4,096 scores, and then a logistic model was used to guess people's sexual orientation from the these scores.

I employed 20 -fold cross-validation in training the classifier, which combined the singular value decomposition and logistic regression model. One concern might be why the data were not split using more common methods in machine learning such as the hold-out validation. In hold-out validation, data are split into training and testing sets only once. Since the sample size here is small compared to other machine learning studies (e.g., $\mathrm{n}>1$ million), the hold-out or unseen set might not be representative enough of the underlying distribution of the dataset, and thus have small power. For example, for men, the unseen test set would contain only 256 individuals if a 19:1 split was applied only one time. Repeating the validation 20 times using different combinations of the data would theoretically maximize the power of the study.

Another note is that the dataset contained multiple images for most individuals (see Table 1). Thus, all images of the same individual were assigned to one and only one cross-validation partition. In other words, if an individual has multiple facial images, the images never appeared in both the training and testing partitions in any crossvalidation folds. More details on how this is programmatically implemented is shown in https://osf.io/q39py/. The resulting sample size by cross-validation fold, train-test partition, and number of images is reported in Table S2 of the Supplementary Materials.

A related question is why the data were not split into training, validation, and testing sets. ${ }^{3}$ This is because, as mentioned earlier, the study employed a pre-trained

\footnotetext{
${ }^{3}$ Typically, machine learning studies split the data into training and testing (i.e., unseen) sets. The training set would be further split into training and validation sets. The training sets would be used to train algorithms with different hyper-parameter settings. The validation sets would then be used to evaluate the models. After a best model was selected, the testing set would be used to evaluate the generalizability.
} 
algorithm (VGG-Face), and there was no hyper-parameter tuning or no model selection for the singular value decomposition and logistic regression models. Therefore, splitting the data further into training-validation sets was unnecessary (for a review on validation and model-selection methods, see Raschka, 2020).

Image Augmentations. To test the influence of image background on the sexual orientation algorithm, I applied a rectangular mask to the center of each facial image. The entire dataset of masked images was then used to classify sexual orientations using the same 20 -fold cross-validated model pipeline described above. A total of 29 augmentations using masks of increasing sizes was conducted. In the first augmentation, $3.3 \%$ (100\% divided by 30 augmentations) of the image was masked in terms of the image area and a classification score was recorded. In the last augmentation, $96.7 \%$ of the image was masked. Only a very tiny border, made up of $3.3 \%$ of the entire area, remained on the image. Additionally, I conducted an augmentation using a mask that covered $100 \%$ of the image to produce a random classification. When the image was entirely masked, the algorithm generated a random classification power. In this case, I verified that the VGG-Face algorithm produced facial scores of 4,096 zeros. These scores produced zero-only predictions, which finally resulted in $\mathrm{AUC}=0.500(95 \% \mathrm{Cl}=[0.50,0.50])$. The first row of Figure $\mathrm{S} 1$ in Supplementary Materials provides a few examples of the image augmentations.

\section{Results}

Sexual Orientation Classification. I first report results about the extent to which people's sexual orientation can be detected from their images, parallel to the main results of Wang \& Kosinski (2018). The AUC results and their confidence intervals are 
reported in Figure 3. Unlike Study 1a and 1b, I report AUC results separately according to the number of facial images to replicate the format reported in Figure 2 of Wang and Kosinski (2018). I also report the average AUC score, which was calculated by averaging the classification scores across facial images within each participant. Corresponding confusion matrices that indicate the accuracy of the model's classification are reported in Figure 4. Other common evaluation metrics are reported in Table 4.

The model's average AUC was $.702(95 \% \mathrm{Cl}=[.692, .712])$ for women and .662 $(95 \% \mathrm{Cl}=[.647, .677])$ for men. AUC increased for both women and men when more facial images were used per person. For five facial images, the AUC increased to .732 $(95 \% \mathrm{Cl}=[.691, .774])$ for women and $.797(95 \% \mathrm{Cl}=[.736, .858])$ for men. This AUC was similar to those found by Wang and Kosinski (2018) for women, however for men, this AUC was lower than Wang and Kosinski's AUC of around .81 when one image was used per person. Overall, the above results confirmed that it is possible to detect sexual orientation from images posted on the dating website. Next, I examined how masking the facial images affects the model's classification rate to assess the likely impact of a self-presentational feature on sexual orientation detection.

\section{Figure 3.}

Baseline AUC Results by Number of Images and Gender for Study $1 \mathrm{C}$ 


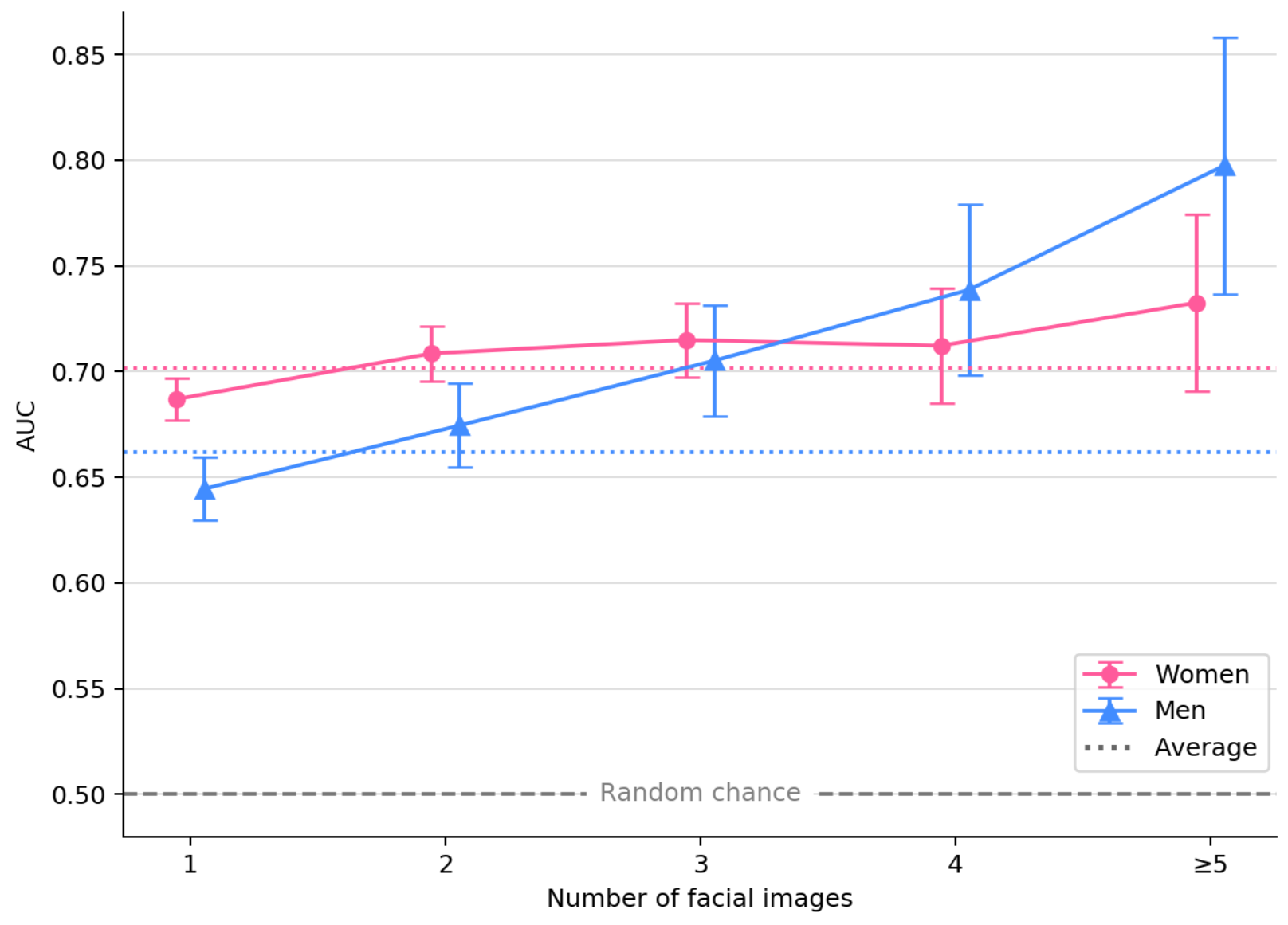

\section{Figure 4.}

Baseline Confusion Matrices by Number of Images and Gender for Study $1 \mathrm{c}$

Women: 

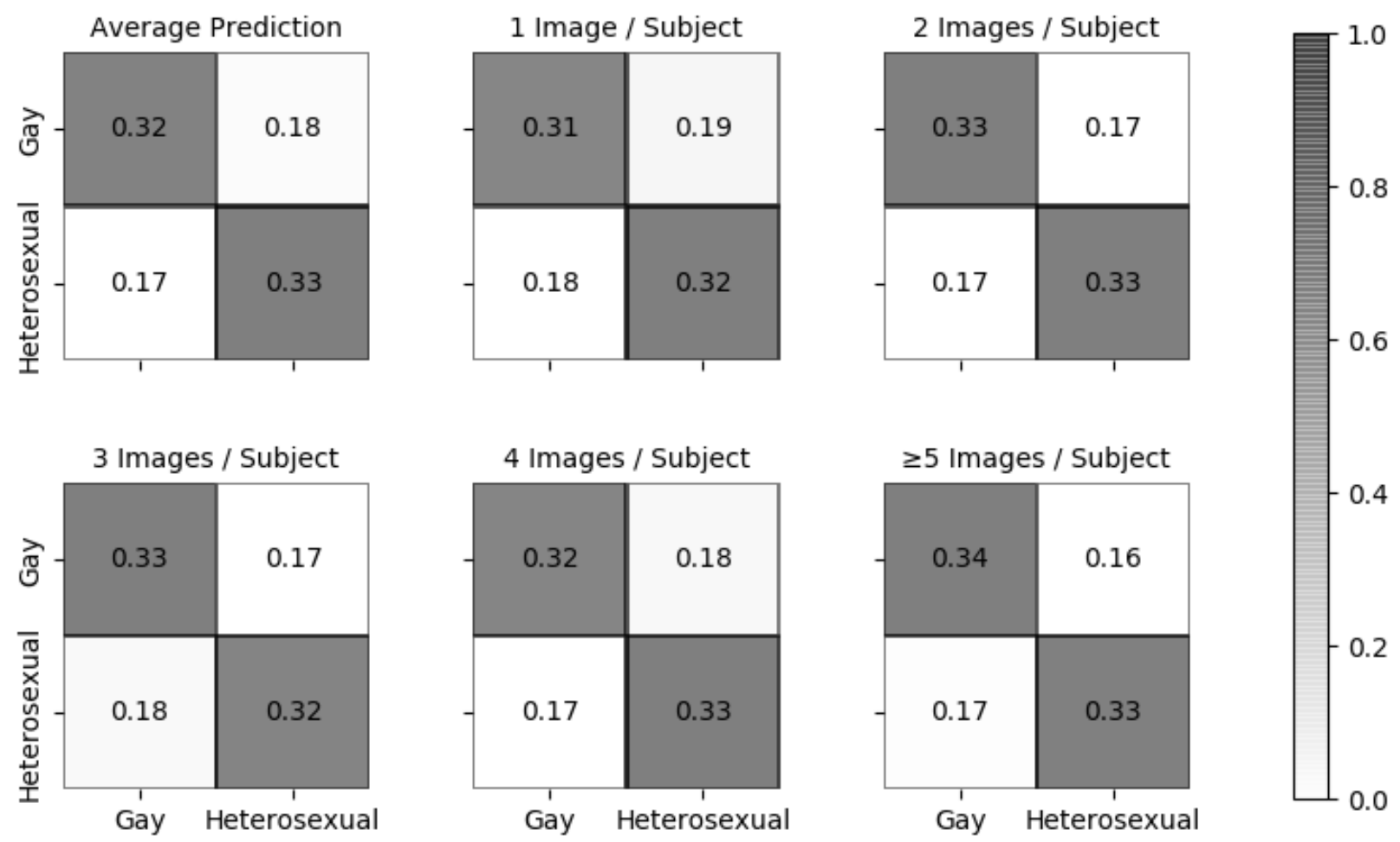

\section{Men:}
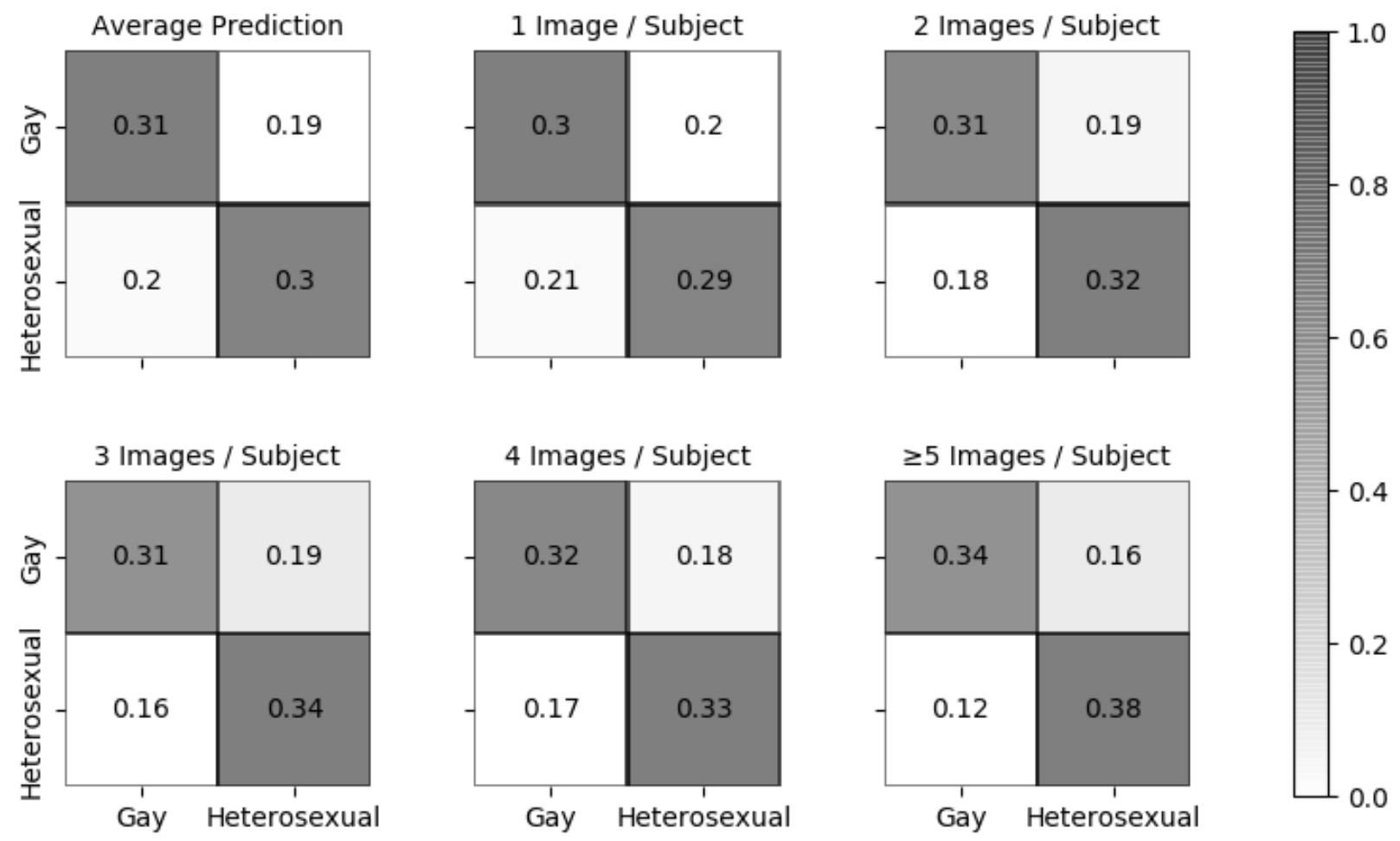

Table 4. 
Baseline Accuracy Results by Number of Images and Gender for Study $1 \mathrm{c}$

\begin{tabular}{|c|c|c|c|c|c|c|c|c|}
\hline & \multicolumn{4}{|c|}{ Women } & \multicolumn{4}{|c|}{ Men } \\
\hline & Accuracy & Precision & Recall & F1 & Accuracy & Precision & Recall & F1 \\
\hline Average & $64.83 \%$ & $64.72 \%$ & $65.18 \%$ & $64.95 \%$ & $61.10 \%$ & $61.21 \%$ & $60.66 \%$ & $60.93 \%$ \\
\hline 1 Image & $63.64 \%$ & $63.39 \%$ & $64.55 \%$ & $63.96 \%$ & $59.37 \%$ & $59.44 \%$ & $58.98 \%$ & $59.21 \%$ \\
\hline 2 Images & $65.22 \%$ & $65.23 \%$ & $65.17 \%$ & $65.20 \%$ & $62.56 \%$ & $62.31 \%$ & $63.58 \%$ & $62.94 \%$ \\
\hline 3 Images & $65.62 \%$ & $65.83 \%$ & $64.98 \%$ & $65.40 \%$ & $64.75 \%$ & $64.03 \%$ & $67.35 \%$ & $65.65 \%$ \\
\hline 4 Images & $65.68 \%$ & $65.40 \%$ & $66.57 \%$ & $65.98 \%$ & $65.29 \%$ & $64.91 \%$ & $66.55 \%$ & $65.72 \%$ \\
\hline$\geq 5$ Images & $66.85 \%$ & $66.91 \%$ & $66.67 \%$ & $66.79 \%$ & $72.28 \%$ & $70.64 \%$ & $76.24 \%$ & $73.33 \%$ \\
\hline
\end{tabular}

Image Augmentations. Figure 5 shows the AUC results when different proportions of the facial image have been masked. For ease of interpretation and brevity in the main text, I report the AUC scores averaging across the predictions from the varying levels of facial images per participant. Table S3 of the Supplementary Materials reports the AUC scores and significance tests against random classifications. For compatibility with previous research, I also report AUC scores generated using only one image per person in Table S4 of the Supplementary Materials. Table S5 and S6 of the Supplementary Materials report other common evaluation metrics using averaged predictions or only one prediction respectively. All results were reported separately for women and men.

Of the 29 degrees of masking, no AUC dropped to chance level (AUC $=0.50$ ) for both women and men. All AUCs were significantly higher than random chance ( $p$ $<.001)$. As seen in Figure 5, AUC scores started to drop when masks were applied to each facial image. Nevertheless, these scores remained above random chance throughout. When the face was effectively masked in each facial image (mask area = $50 \%$ of the entire image), the AUC scores for gay vs. heterosexual people translated to Cohen's $d=2.295$ for women and $d=1.631$ for men, which are huge effect sizes (Sawilowsky, 2009). As I applied larger masks, the AUC continued to degrade but never 
reached random-chance level. At the most extreme case, when $96.7 \%$ of the image was masked, AUC scores for both women and men were above random-chance level and translated into Cohen's $d=0.760$ for women and $d=1.072$ for men (on average large effect sizes).

Figure 5.

AUC Results by Different Degrees of Masking Compared to AUC of Random Classifications (Fully Masked Images) for Study $1 \mathrm{c}$
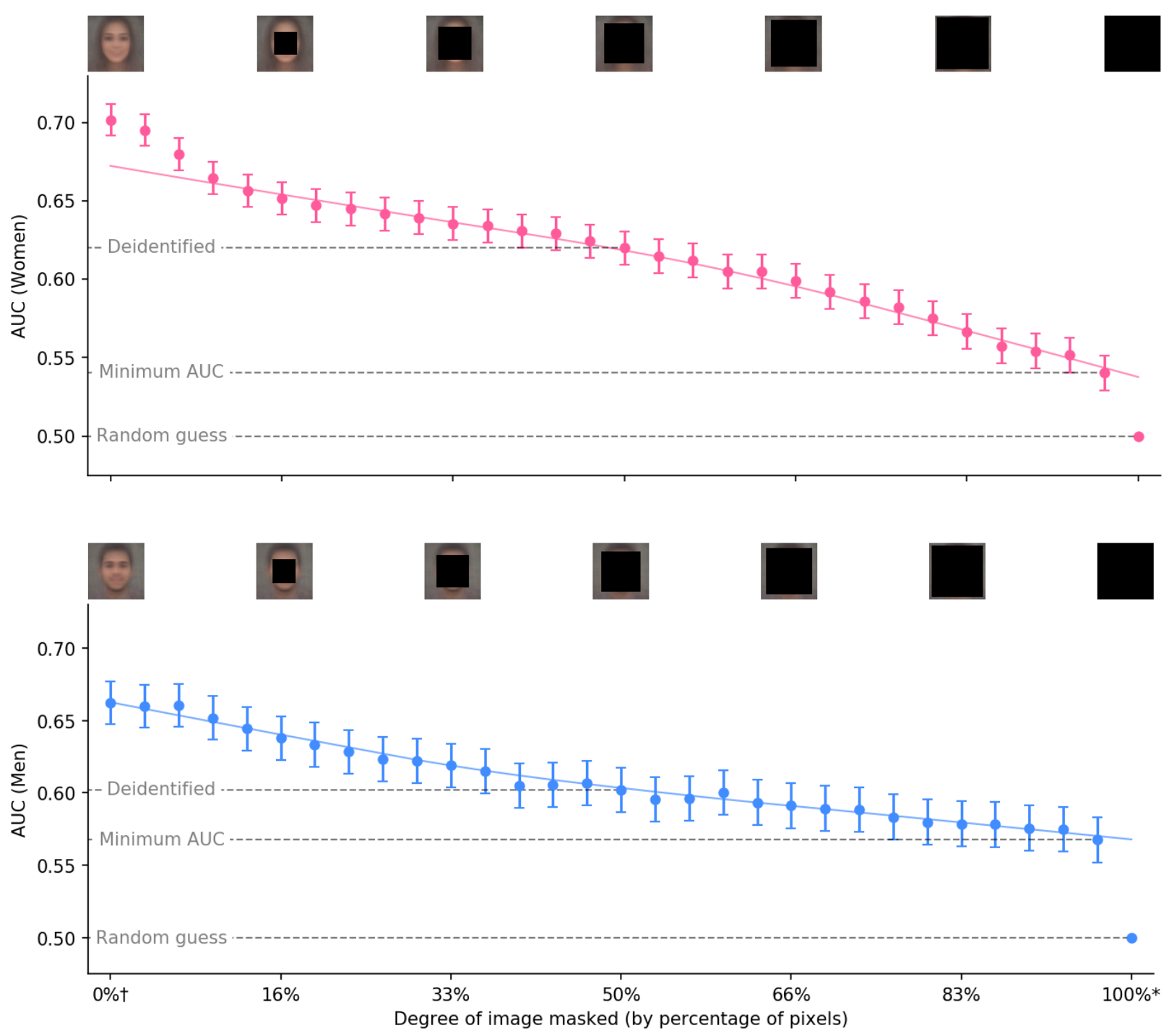

Note: † baseline AUC. * random AUC. 
While AUC scores degraded as images were progressively masked, the degradation did not follow the same magnitude of degradation for image pixels. One interesting finding here relates to how AUC degradation differed by gender; AUC degraded more severely at the beginning for women compared to men. The degradation started to show signs of flattening but picked up again towards the end. However, the degradation pattern followed an almost curvilinear pattern for men, where magnitude of degradation started to drop towards the end for men. For women, the results suggest that the facial regions might have contributed more compared to background, and vice versa for men. This seems to be consistent with the findings in Study 1a, where women on average displayed larger differences in facial attributes between sexual orientations.

\section{Discussion}

Study $1 \mathrm{c}$ demonstrated that sexual orientation classification was possible using the dataset I collected, replicating the results in Wang and Kosinski (2018). Study 1c also found that the image background or pixels at the image border contributed to sexual orientation classification. These findings were alarming because in the most extreme case, masked images contained only $3.3 \%$ of the original pixels. Yet, these pixels could generate above-random-chance classifications. When interpreted together with the findings in Study $1 \mathrm{a}$ and $1 \mathrm{~b}$, these findings suggest it is possible that gay vs. heterosexual people chose to upload photographs using different self-presentational facial attributes as well as different backgrounds on the online dating website. The differences in turn allowed the model to classify their sexual orientation using these selfpresentational features. Importantly, these findings also warn about a risk to privacy 
loss that was previously underestimated, because masking the face might not effectively prevent the exposure of sexual orientation in facial images, contrary to previous research in privacy protection (Zhang et al., 2014).

\section{Study 2a: Brightness Differences in Facial Images across Sexual Orientations and Facial Image Regions}

The goal of this study was to assess whether the overall brightness of the image was yet another self-presentational variable that could reveal people's sexual orientation. Previous research found that gay men's average faces had brighter skin tones (Wang and Kosinski, 2018). However, it could be possible that skin tones were related to the overall brightness or illumination of the images. To test this, I separated the image into facial region ( $50 \%$ of the image area) and image background ( $50 \%$ of the image area). I extracted the average brightness of these regions, and examined the brightness by regions and sexual orientation. If the brightness of the facial regions varied according to sexual orientation in the same direction and magnitude with background, it would be likely that the appearance of skin tone was related to the overall brightness or illumination of the image. In this case, the difference in the general brightness of the image would suggest a possible form of self-presentation, where people in the sample chose to present themselves in brighter or darker images or locations that were illuminated differently (e.g., indoors vs. outdoors) according to their sexual orientation.

\section{Methods}

Facial Images. I employed the same dataset of facial images as Studies 1a, 1b and $1 \mathrm{c}$. 
Image Regions. To separate the facial image into different regions, I designed a fixed mask using the average facial landmarks extracted from each facial image. Since all faces were aligned at the same location, a fixed mask would be reasonably effective in covering all faces (or backgrounds) in the images. Figure 6 shows an example of the masks used to separate the regions. The size and shape of the mask were optimized such that the mask only occupied $50 \%$ of the entire image, at the same time covering a slightly larger area of the average facial region (see average facial landmarks in Figure 6). I also ensured that the mask would cover all the skin area including the neck. To extract the background regions, I blocked the facial region using the mask (see Figure 6 , left panel). To extract the facial regions, I inverted the mask and blocked the background regions (see Figure 6, right panel). I applied the standard and inverted mask onto every facial image to produce two masked images for each original facial image. As the number of facial images per participant is different, I created a composite image for each person.

\section{Figure 6.}

An Example of the Facial Mask Used to Separate the Facial Regions for Study 2a 
Facial Region Masked

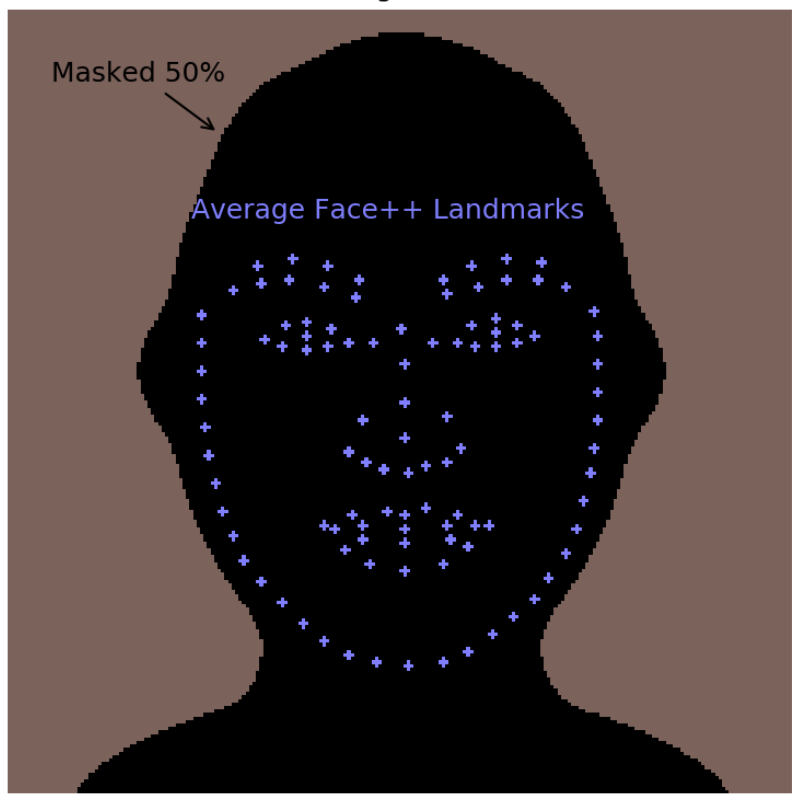

Image Background Masked

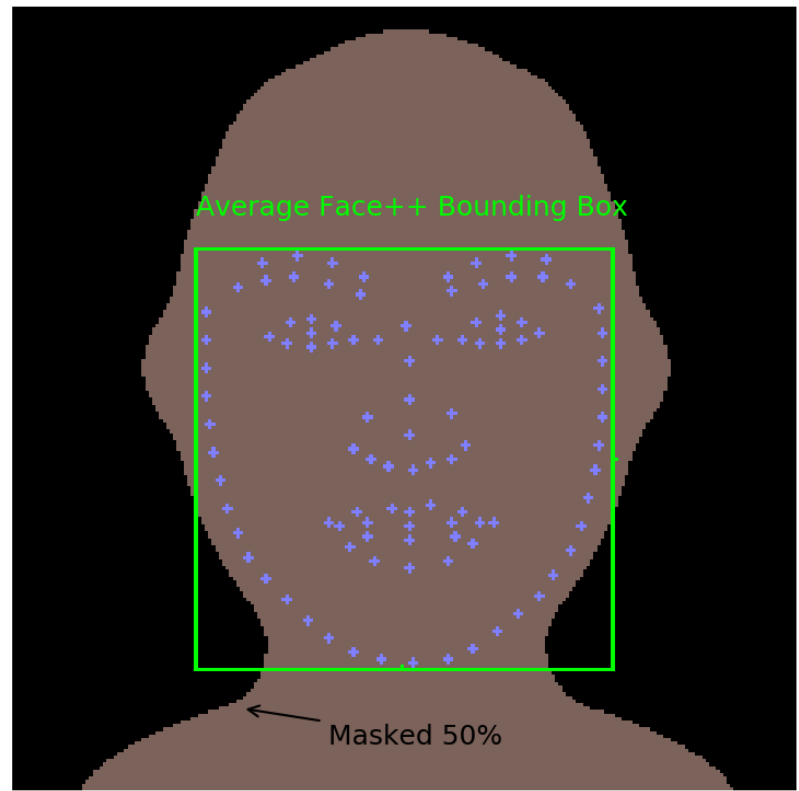

Image Brightness. Image brightness was measured by the average value of all pixels in the image for each color channel. The higher the value of the pixel, the brighter the image. Since pixels are valued from 0 to 255 , I normalized them to a range of 0 to 1 . I extracted two within-person brightness measures, i.e., the brightness of the background and that of the facial region for each participant.

\section{Results}

Figure 7 plots the image brightness by image regions and sexual orientation separately for each gender. Table S7 in Supplementary Materials reports the statistical tests on the differences in brightness for each color channel by image regions and sexual orientations. Note that the general trend in how brightness varied was similar across each color channel (red, green, and blue). This meant that the difference was indeed due to brightness and not hue (see Table S7 in Supplementary Materials). Looking at Figure 7, the facial regions were across the board brighter than the background, which confirms that the masks correctly separated the facial regions and 
the background. A significant difference in image brightness was observed between gay and heterosexual people in the sample for both genders and facial regions.

\section{Figure 7.}

Image Brightness by Sexual Orientation, Facial Regions, and Gender for Study 2a
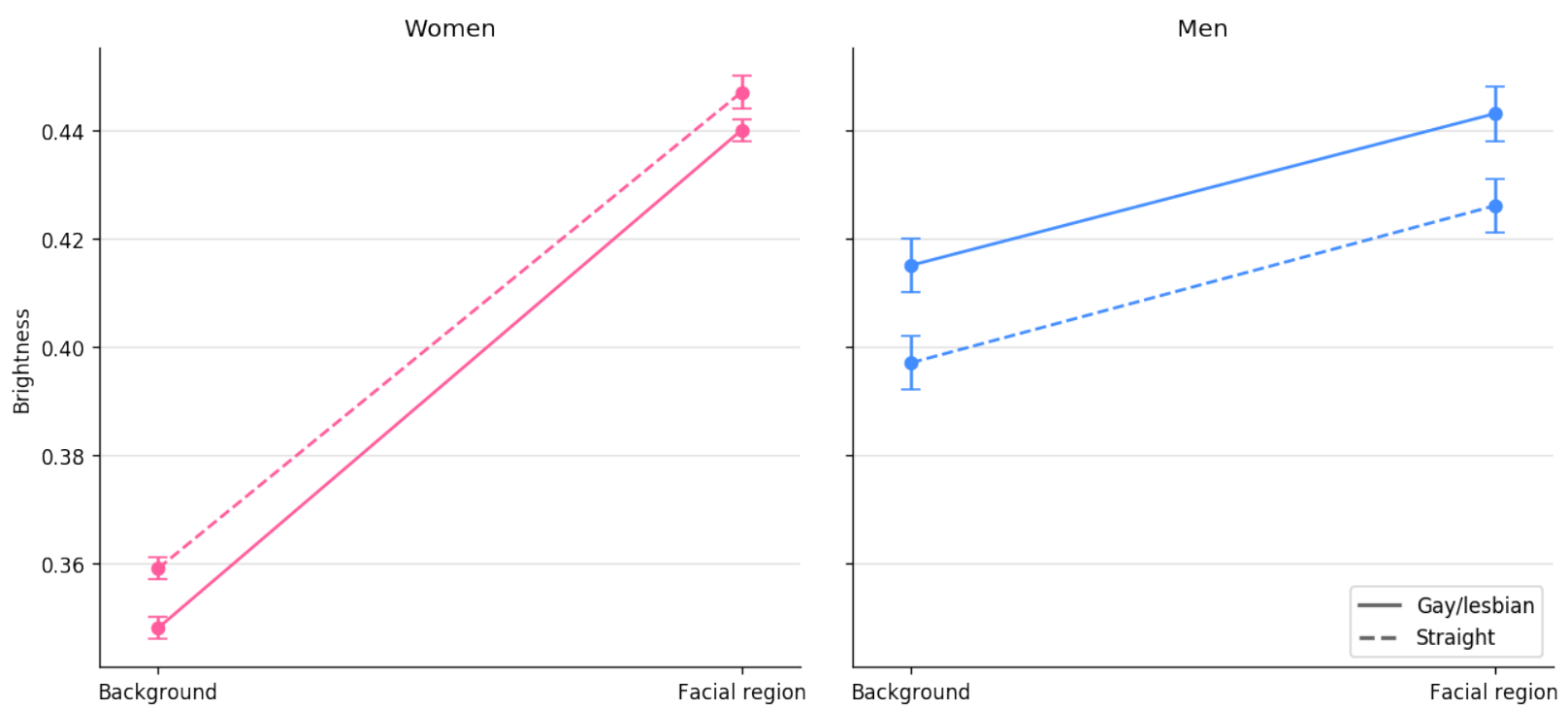

On average, images were significantly darker for lesbian women compared to heterosexual women $(p<.001)$, whereas images were significantly brighter for gay men compared to heterosexual men $(p<.001)$. Comparing the regions, image background was on average darker than the facial region, but the magnitude and direction of the variations were similar. In other words, if facial regions were on average darker for a given sexual orientation, the background region would also be darker for that sexual orientation compared to the other group. This supports the hypothesis that skin tone differences across sexual orientations was related to differences in the overall brightness of the image and not just the facial regions. This suggests a form of selfpresentation, where people preferred different image brightness or illumination according to their sexual orientations. 
I further conducted a mixed-design ANOVA separately for each gender. There was a significant main effect of masked regions on brightness differences for both women $(F(1,10338)=4711.06, p=<.001, n p 2=.313)$ and $\operatorname{men}(F(1,5122)=199.14$, $\mathrm{p}=<.001, \mathrm{np} 2=.037)$. There was also a significant main effect of sexual orientation on brightness differences for both women $(F(1,10338)=19.32, p=<.001, \eta p 2=.002)$ and men $(F(1,5122)=42.22, p=<.001, n p 2=.008)$. However, I did not observe any significant interaction between masked regions and sexual orientation in terms of brightness difference for both women $(F(1,10338)=2.64, p=.104, \eta p 2=<.001)$ and men $(F(1,5122)=0.037, p=.847, n p 2=<.001)$.

\section{Discussion}

Study $2 a$ found that brightness in the facial region varied together with background region according to sexual orientation. For example, gay men on average uploaded facial images that are brighter in both the facial and background region compared to heterosexual men. The brighter skin tone observed in the previous study (Wang and Kosinski, 2018) for gay men might have been due to gay men taking and uploading generally brighter or more illuminated facial images to the dating website. In other words, the brighter images for gay men might have made their skin appear brighter compared to heterosexual men. Overall, this difference in image brightness suggests a coherent story of how gay and heterosexual people in the sample chose to upload different facial images according to their self-presentational preferences.

The difference in illumination of facial images across sexual orientation and gender groups might be attributed to many reasons. One interpretation might be the use of camera flash lights. However, flash lights should be directed to the face, and should 
not result in the overall brightness of the image. Another possible interpretation might be the fact that people used different photo-editing processes, such as applying image filters (Haferkamp et al., 2012; Hancock \& Toma, 2009; Ota \& Nakano, 2021). A third interpretation might come from theory in person-environment transactions, which posits that people actively choose their daily environments according to their dispositions (Matz \& Harari, 2021; Wrzus et al., 2016). It would be possible that for people with brighter images, the images were taken outdoors. This interpretation might also help to explain why image background in Study 1c contributed to sexual orientation classifications for men. That said, it might be possible that a combination of factors, i.e., flash lights, filters, locations, and even skin tones, contributed to the observed differences, and these factors might play out differently depending on the gender.

\section{Study 2b: Testing How Image Brightness Contributed to Sexual Orientation Classifications}

Study $2 b$ tests whether differences in image brightness played a significant role in classification of sexual orientation. To do so, Study $2 \mathrm{~b}$ employed an image augmentation technique: blurring. I blurred the images progressively and tested the classification power of blurred images using the sexual orientation classifier. When images were completely blurred, only brightness differences remained. This helped me to measure the contribution of brightness on classification of sexual orientation. If three values that measured the image brightness for each color channel can classify sexual orientation, the classifications are likely attributed to self-presentation.

Importantly, Study $2 \mathrm{~b}$ was also designed to evaluate the limit of privacy protection in sexual orientation classification. Image blurring is a frequently used 
strategy to de-identify facial images and to protect the privacy of individuals (Li et al., 2017). Augmenting the image by blurring aims to test whether this privacy-protection strategy may prevent the loss of private information. If blurring were effective, sexual orientation classification using blurred images should drop to chance level. If not, it would reveal a threat to privacy loss and a limit to privacy protection.

\section{Methods}

Facial Images. I employed the same dataset of facial images as Studies 1a, 1b, $1 \mathrm{c}$ and $2 \mathrm{a}$.

Image Augmentations. The second row of Figure S1 in Supplementary Materials provides a few examples of the image augmentations. I blurred each image by downsizing it to a target width and height. The downsizing algorithm interpolated every new pixel using the average values of its respective source pixels. ${ }^{4}$ As the algorithm employed in this study requires images of size 224 by 224 pixels, I enlarged every image back to its original size after it was downsized.

The entire dataset of blurred images was used to classify sexual orientations following the same 20 -fold cross-validated model pipeline described in Study 1c. A total of 29 augmentations using target width of decreasing sizes was conducted. Figure S2 in Supplementary Materials provides a plot of the target width by augmentation number. In the first augmentation, a target width of 112 pixels was used (50\% of the original image width) and a classification score was produced. This step was repeated until, in the last augmentation, the image was downsized to only one pixel. In other words, it was completely blurred.

\footnotetext{
${ }^{4}$ For example, if a 224 by 224 image were downsized to one pixel, the resulting image would contain the average pixel values of the original image.
} 
To evaluate these classifications, I compared them to random classifications. Unlike Study 1c, random classifications were generated using the downsized average image of the entire dataset (separately for each gender). As expected, blurred average image of the entire dataset generated a random classification score of $A U C=0.500$ $(95 \% \mathrm{Cl}=[0.50,0.50])^{5}$ for each gender.

\section{Results}

Figure 8 shows the average AUC results with confidence intervals. Table S8 of the Supplementary Materials reports the average AUC results and significance test against random classifications. Table S9 of the Supplementary Materials reports AUC scores generated using one image per person. Table S10 and S11 of the Supplementary Materials report other common evaluation metrics using averaged predictions or only one prediction respectively. All results were reported separately for women and men.

Of the 29 degrees of blurring, no AUC dropped to chance level (i.e., AUC $=0.50$ ) for both women and men. All AUCs were significantly higher than random chance ( $p$ $<.001)$. As seen in Figure 8, slightly blurring the facial images did not lead to any decrease in AUC scores for both women and men (i.e., for the first four augmentations). In fact, AUC scores even increased for men when facial images were blurred to 44 by 44 pixels. This could be due to blurring eliminating noisy pixels, thus allowing the algorithm to "focus" better on the useful pixels.

As I increased the degree of blurring, the AUC scores remained high for both women and men but started to decrease until the midpoint of the augmentations. At this

\footnotetext{
${ }^{5}$ In this case, VGG-Face algorithm produced random facial scores (mostly zeros), but when they entered the 20 -fold cross-validated logistic regression, they yielded zero-only classifications like Study $1 \mathrm{c}$.
} 
point, the facial features started to become too blurred to be recognized, as every facial image was downsized to only 16 by 16 pixels. Despite this, the AUC scores remained high at $A \cup C=.62$ for women and $A U C=.59$ for men. These scores translated to Cohen's $d=2.36$ for women and $d=1.50$ for men, which are on average huge effect sizes. As the blurring went along further, AUC started to degrade more for women but remained almost constant for men. Finally, at the most extreme case, i.e., when images were downsized to one pixel, AUC scores for both women and men were above chance level and translated into Cohen's $d=0.55$ for women and $d=1.00$ for men (i.e., large effect sizes).

Figure 8.

AUC Results by Different Degrees of Blurring Compared to AUC of Random (One-pixel Blurred Image of Dataset) for Study $2 b$

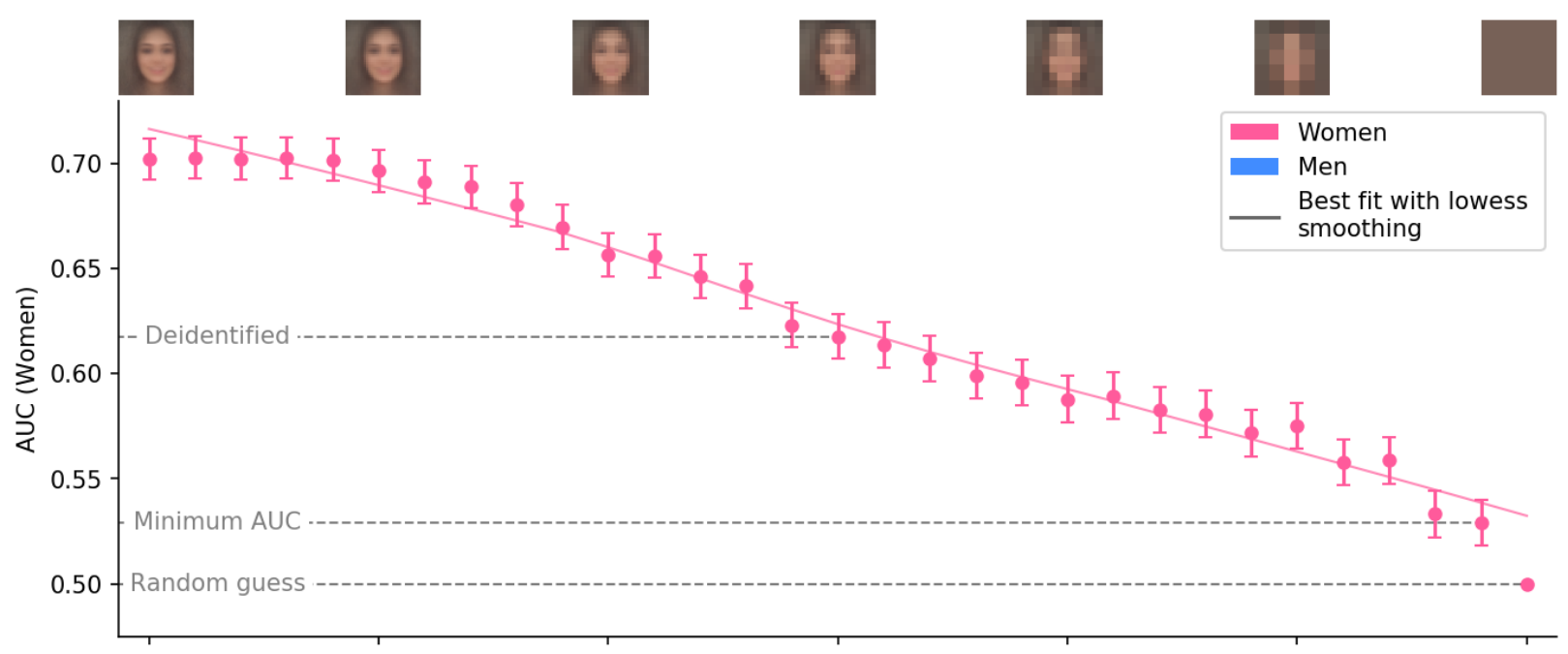




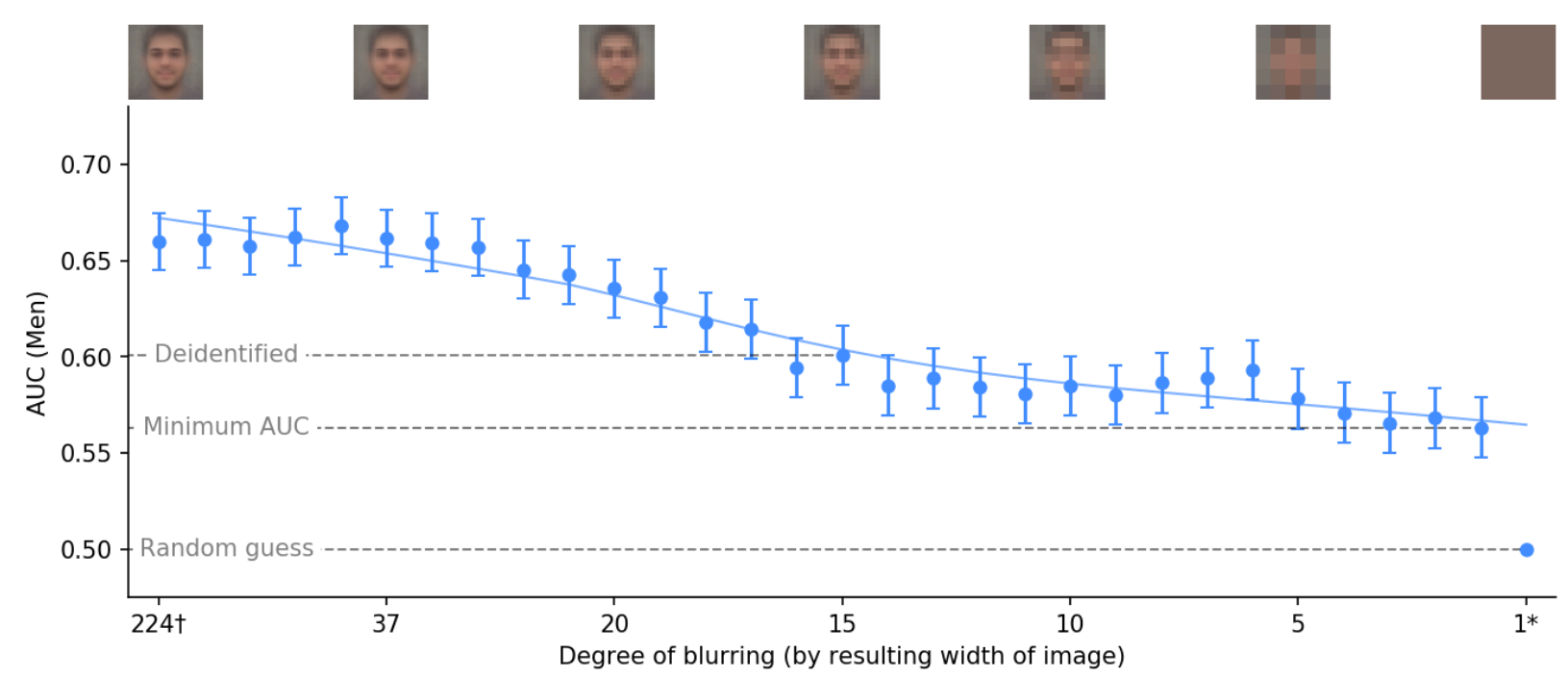

Note: † baseline AUC. * random AUC.

Looking at the overall AUC degradation pattern, some obvious differences across genders were observed, as in Study 1c. AUC scores degraded as pixels were progressively blurred, but the decrease in scores did not follow the same magnitude of degradation in image quality. AUC degradation seemed to follow a linear pattern for women but a slightly curvilinear or even somewhat random pattern for men. Intriguingly, for men, the degradation started to show signs of increase beyond the point where facial features became unrecognizable (when downsized to 6 by 6 pixels) compared to earlier augmentations. It is interesting that this general trend for both genders was somewhat consistent with Study 1c, where the AUC degradation was slightly more severe for women but not so for men.

\section{Discussion}

These results, when interpreted together with those in Study 1c, might provide a consistent story about how men chose photographs with different backgrounds and image lighting (possibly due to different locations or ambient environments). Finally, like Study $1 \mathrm{c}$, these results demonstrated a serious threat to privacy. This is reflected in the 
most extreme case, where the only information necessary for classifications could be as few as three numbers representing the brightness values of each color channel.

\section{General Discussion}

This research examined whether gay and heterosexual people presented themselves differently in facial images that were uploaded to a dating website, and whether these differences contributed to classification of sexual orientation. Study 1a showed that self-presentational facial attributes extracted from photographs differed significantly across sexual orientations. Study $1 \mathrm{~b}$ showed that these differences contributed to classification of sexual orientation. Study $1 \mathrm{c}$ replicated the sexual orientation classifier in Wang and Kosinski (2018), and examined the contribution of image background on sexual orientation classification. The results showed that the classifier was influenced by the image background, and that even a $97 \%$ masked facial image could classify sexual orientation at rates higher than random chance. Several robustness checks revealed that this pattern was not due to artefacts in the algorithm. Study 2a examined another self-presentational feature: image brightness. Results show that the difference in skin tone across sexual orientation was due to overall brightness or illumination of the image. Given that brightness varied across sexual orientation, Study $2 b$ tested whether highly blurred images, where only brightness information remained, would classify sexual orientation. As expected, results showed that image brightness contributed to classification of sexual orientation, particularly for men. These results demonstrate that people presented themselves differently in facial images, classification of sexual orientation was influenced by these differences, and the risk of privacy loss might be higher because de-identification strategies were ineffective. 
Theoretical Contribution. If self-presentation mattered for classification of sexual orientation, what about facial morphology? Wang and Kosinski (2018) acknowledged the contribution of both morphology and self-presentation but framed their study using the Prenatal Hormonal Theory. For example, they wrote, "Some of the differences between gay and heterosexual individuals, such as the shape of the nose or jaw, are most likely driven by developmental factors." (p. 254). My study aimed to provide an alternate narrative by showing that self-presentation makes a significant contribution to sexual orientation classification. For example, 12 self-presentational facial attributes could classify sexual orientation at AUC scores almost comparable to when the entire facial image were used (4,096 VGG-scores) for women.

One question that might arise is which of these influences, facial morphology or self-presentation, contributed most to classification of sexual orientation. There is ample evidence in facial perception research pointing to confounds in facial images, and how these confounds might render photographs unreliable measures of facial identity, appearance, or morphology (Jenkins \& Burton, 2011; Jenkins et al., 2011). For example, Jenkins et al. (2011) found that within-person variability in photographs is sometimes even larger than between-person variability, which caused unfamiliar raters to misidentify faces in the photographs. Noyes and Jenkins (2017) found that camerato-person distance can also moderate the person's face shape in photographs, leading to different levels of face recognition by humans. Consistent with this line of research, my study would argue that my sample, i.e., dating photographs, is not fit to answer morphological related questions. It would be likely that facial morphology and self- 
presentation both contributed to classification of sexual orientation, and there might be a significant interaction between the two.

Future research interested in the morphological contribution of sexual orientation classification should consider using standardized facial images that are taken in controlled conditions (such as the lab). Future studies should also control for the influence of important self-presentational features such as the presence of glasses and image backgrounds. In particular, social cognition researchers typically use stimuli in which all non-facial parts of the face (including hair) are occluded (see Oosterhof \& Todorov 2008, Figure 1, for example). Researchers can use such controlled, standardized stimuli in which there is no room for self-presentation to determine whether sexual orientation can be detected from morphology.

These arguments could be easily dismissed on grounds that advanced facial recognition algorithms are less affected by self-presentational features (Phillips, 2017; Prasad et al., 2020; Taigman et al., 2015). For example, the VGG-Face algorithm employed in this study was developed using 2.6 million facial images of 2,622 unique individuals (Parkhi et al., 2015; Phillips, 2017; Prasad et al., 2020). Given that, on average, 1,000 images per person were fed into the algorithm, researchers believed it would start to recognize high-level facial patterns, such as the contour of the jaw or the shape of the nose and ignore confounding features such as the image background. However, consider the following illustration. Diane is an extrovert. She enjoys outdoor activities and likes taking selfies of herself outdoors. Mary is an introvert. She likes to stay at home and rarely takes photographs of herself. When they uploaded photographs to their social media platforms, Diane consistently chose photographs of her outdoors 
while Mary chose only a picture of her in her bedroom. Therefore, their personality traits, such as whether they are extroverted, might be consistently reflected in their selfpresentational behaviors, e.g., lighting of their photographs, skin tan, and image background, but not so much in their facial morphology or appearance.

My findings suggest that facial recognition algorithms might not have eliminated self-presentation features. While facial recognition algorithms perform well under different lighting conditions (Phillips, 2017), it does not mean that differences in lighting or brightness, a form of self-presentational feature as seen in Studies $2 a$ and $2 b$, are disregarded by the algorithm. The findings in Studies $2 a$ and $2 b$ suggest that when the algorithm was applied to sexual orientation classification (from facial recognition), it started to rely on such features. Thus, future research interested in facial morphology, should consider methods in other areas of machine learning. For example, 3D face reconstructions using multiple facial images of the same person (Gecer et al., 2019; Tran et al., 2018) might produce more accurate morphological models, might be less affected by self-presentational features, and might more robustly model the relationship between morphology and behavioral outcomes (Kittler et al., 2016).

In terms of impression management, the findings in this research are consistent with the idea that people present themselves differently based on their values, goals, identity, and social contexts (Goffman, 1959; Leary \& Kowalski, 1990; Schlenker, 2012). My study found that people in the sample systematically uploaded different photographs to the dating website according to their sexual orientation and gender. For example, men on average uploaded photographs that differed significantly in image background and brightness across sexual orientations. As elucidated, while self-presentational 
differences were observed, impression management research suggests that selfpresentations can be either conscious or subconscious (Leary \& Kowalski, 1990). It is beyond the scope of my study to further examine the motivations.

The findings here also contribute to facial perception research. The literature has established a vast framework showing how morphological and self-presentational differences influence social evaluations (Oosterhof \& Todorov, 2008, 2009; Todorov, 2008; Todorov et al., 2008). However, it seems there is a scarcity of research examining how image backgrounds or lighting in naturalistic facial images affect perceptions of faces. Future facial perception research can explore whether image properties affect the impressions of people, and how properties interact with morphologies or other selfpresentational styles in forming facial perceptions. The findings here also seemed to connect to the burgeoning literature around the biasing nature of faces (Olivola et al., 2014; Olivola \& Todorov, 2010; Todorov et al., 2015). If perceptions of faces can be biased, how do different self-presentations identified in this study affect or improve the accuracy of these perceptions? Future studies at the intersection of impression management and facial perception research could perhaps explore these questions further.

Finally, my study might relate to research in person-environment transactions (Matz \& Harari, 2021; Wrzus et al., 2016). When correlating naturalistic facial images with behaviors, it is often impossible to eliminate the influence of image background. In fact, the findings here might demonstrate what was shown in Torralba et al. (2008), that scenes can be consistently predicted using tiny images. I did not directly test whether the difference in background and lighting was caused by ambient environmental 
conditions (or location), but if these conditions influenced the results here, it would open new doors to research. Perhaps future research can evaluate whether people of different characteristics or personality traits systematically take selfies in different locations, whether these locations are correlated with other self-presentational styles, and how these locations further influence people's psychological states.

Practical Implication. Most importantly, my research revealed a danger to privacy loss that was underestimated in previous research. The results here suggest that a small number of pixels at the image border or even one pixel representing the average brightness of the image can expose sexual orientation information. Therefore, masking or blurring the face in naturalistic facial images does not completely prevent the loss of this sensitive information. One counter-argument is that classification of sexual orientation using self-presentational differences might be easier in the context of dating but more difficult in other contexts. However, previous research might suggest that some self-presentational behaviors are habitual or consistent, thus do translate to other contexts (Agüera y Arcas et al., 2018). For example, gay individuals reported that they wore glasses more (and preferred the aesthetics of doing so) compared to heterosexual individuals. Therefore, "non-transient" self-presentational factors might consistently reflect in other contexts, such as job interviews, and expose sensitive information.

We live in a day when we are closely connected to the digital world and acculturated to expressing ourselves through online platforms. However, companies such as Clearview Al are taking advantage of this trend by amassing huge databases of facial images without our consent, and training facial recognition algorithms to expose 
our sensitive information in the name of law enforcement (Rezende, 2020). While research suggests that consumers can employ do-it-yourself methods to protect themselves (Li et al., 2017; Matzner et al., 2016; Shan et al., 2020; Zhang et al., 2014), the findings here indicate that these methods might not be as effective as researchers imagined. If governments and companies do not take the necessary steps to address this matter, the alternative to privacy protection would be people uploading fewer photographs and engaging in less online activity. However, this would entail a tradeoff between privacy and freedom of expression, one that would be detrimental to our society. Overall, based on the results in this study, I recommend against shifting the burden of privacy protection to consumers. Fortunately, some governments have started to limit the use of certain facial recognition systems (Conger et al., 2019). They are also implementing stricter privacy protection laws (Rothstein \& Tovino, 2019). It is my hope that with this trend continuing, people will not face the privacy concerns addressed in this study.

Limitations. One question regarding Studies $1 \mathrm{c}$ and $2 \mathrm{~b}$ might be whether the pre-trained VGG-Face algorithm assisted the classification when the images were masked or blurred. In other words, were there artefacts in the pipeline or algorithm? To answer this question, I correlated the VGG-scores produced by the image augmentations with the scores produced by the original images. I found that the correlations decreased as images were progressively masked. When the images were fully masked, VGG-Face produced zero-only scores. When fully blurred images of the dataset were entered, VGG-Face produced the same random scores (mostly zeros) for every image. These scores, when fed into the logistic regression model, all produced 
predictions of zero, resulting in $A \cup C=0.50(95 \% \mathrm{Cl}=[0.5,0.5])$. These results show that the VGG-Face algorithm did not assist with the classifications and there were no artefacts in the pipeline. Additionally, I repeated the entire study by custom-training the VGG-Face algorithm (i.e., not using the pre-trained weights) using the augmented images for each of the 29 augmentations. I found that this process produced even higher classification powers, which might indicate over-fitting. As an additional robustness check, I repeated the steps using different validation techniques. The results remained similar. These analyses confirmed that the classification powers reported here were indeed due to background or image brightness and not due to the "robustness" or artefacts of the VGG-Face algorithm.

A related issue might be why image augmentations were used to explain the algorithm in the first place. To elaborate, image augmentation is an attribution technique used in machine learning to increase interpretability (for other methods, see Biecek, 2018). This technique is not new and has been widely applied to many areas of machine learning (Shorten \& Khoshgoftaar, 2019; Zeiler \& Fergus, 2013). However, I deliberately chose image augmentations, i.e., masking or blurring, because they are straightforward and offer results that were easy to interpret. More importantly, the augmentations addressed the research questions in this study. One question was whether the background mattered for classification of sexual orientation. By masking the facial regions of the image, Study $1 \mathrm{c}$ showed that the background contributed to classification of sexual orientation. Another question was whether image brightness contributed to classifications. Thus, Study 4 blurred the image so that only the brightness information was used to classify sexual orientation. Lastly, the most 
important research question was whether data-protection tools would help people protect their private information (Li et al., 2017; Matzner et al., 2016; Shan et al., 2020; Zhang et al., 2014). Image augmentations directly tested whether such strategies were effective. Nevertheless, future studies can employ different attribution techniques and interpret the results here in tandem.

A question regarding the classification results might be why AUC scores are generally lower in my study, especially for men, compared to Wang and Kosinski (2018), despite following that previous study closely in every procedure. First, the lower AUC scores might be due to the smaller sample. The total facial images in my final dataset is 31,762 , whereas total facial images in Wang and Kosinski (2018) is 35,326 . While my study aimed to collect a larger dataset, the preprocessing step pruned more samples compared to the previous study as participants were also matched on the number of facial images (see Table 1). Second, if the lower AUC scores were due to my reliance on a different sample (i.e., a different dating website), the fact that my study replicated the general trend in Wang and Kosinski (2018) shows that classification of sexual orientation is generalizable, providing evidence for even greater privacy concern. A relevant related question might be whether the AUC scores in the most extreme augmentations were too low to warrant any privacy concerns. The augmentations were deliberately exaggerated to examine whether there was a baseline in which AUC would drop to chance-level. In other words, it would be unlikely that images in the wild would contain augmentations that were as severe as those in the 29th step of the augmentations. Even if they did, it is highly likely that companies, such 
as Clearview Al, possess more facial images (in the hundreds) of the same person. These images might be used to produce much higher classification rates.

\section{Conclusion}

This study examined three research questions, namely whether people presented themselves differently in naturalistic facial images according to their sexual orientations, how differences contributed to classification of sexual orientation, and the implications for privacy. The research found that differences in self-presentation across sexual orientation were significant, and that these differences contributed to classifications. Image augmentations found that even when the face was masked or blurred, sexual orientation was classified at rates significantly higher than chance, signaling a risk to privacy that was previously underestimated. Given these findings, I argue that the burden of privacy protection should not be shifted to the consumers, but must be initiated by governments and companies. 


\section{References}

Agüera y Arcas, B., Todorov, A., \& Mitchell, M. (2018, January 11). Do algorithms reveal sexual orientation or just expose our stereotypes? Medium. https://medium.com/@blaisea/do-algorithms-reveal-sexual-orientation-or-justexpose-our-stereotypes-d998fafdf477

Barrett, L. F., Adolphs, R., Marsella, S., Martinez, A. M., \& Pollak, S. D. (2019). Emotional expressions reconsidered: Challenges to inferring emotion from human facial movements. Psychological Science in the Public Interest, 20(1), 168. https://doi.org/10.1177/1529100619832930

Biecek, P. (2018). DALEX: explainers for complex predictive models in R. The Journal of Machine Learning Research, 19(1), 3245-3249.

Conger, K., Fausset, R., \& Kovaleski, S. F. (2019, May 14). San Francisco bans facial recognition technology. New York Times. Retrieved from https://www.nytimes.com/2019/05/14/us/facial-recognition-ban-san-francisco.html

DeLong, E. R., DeLong, D. M., \& Clarke-Pearson, D. L. (1988). Comparing the areas under two or more correlated receiver operating characteristic curves: A nonparametric approach. Biometrics, 44(3), 837-845.

\section{https://doi.org/10.2307/2531595}

Gecer, B., Ploumpis, S., Kotsia, I., \& Zafeiriou, S. (2019). GANFIT: Generative Adversarial Network Fitting for High Fidelity 3D Face Reconstruction. Proceedings of the IEEE/CVF Conference on Computer Vision and Pattern Recognition (CVPR), pp. 1155-1164. 
ative Adversarial Network Fitting for High Fidelity 3D Face CVPR 2019 pa per.html

Goffman, E. (1959). The presentation of self in everyday life. Anchor.

Haferkamp, N., Eimler, S. C., Papadakis, A.-M., \& Kruck, J. V. (2012). Men are from Mars, women are from Venus? Examining gender differences in self-presentation on social networking sites. Cyberpsychology, Behavior, and Social Networking, 15(2), 91-98. https://doi.org/10.1089/cyber.2011.0151

Hancock, J. T., \& Toma, C. L. (2009). Putting your best face forward: The accuracy of online dating photographs. Journal of Communication, 59(2), 367-386. https://doi.org/10.1111/j.1460-2466.2009.01420.x

Jaeger, B., Sleegers, W. W. A., \& Evans, A. M. (2020). Automated classification of demographics from face images: A tutorial and validation. Social and Personality Psychology Compass, 14(3), e12520. https://doi.org/10.1111/spc3.12520

Jenkins, R., \& Burton, A. M. (2011). Stable face representations. Philosophical Transactions of the Royal Society B: Biological Sciences, 366(1571), 1671-1683. https://doi.org/10.1098/rstb.2010.0379

Jenkins, R., White, D., Van Montfort, X., \& Burton, A. M. (2011). Variability in photos of the same face. Cognition, 121(3), 313-323. https://doi.org/10.1016/j.cognition.2011.08.001

Kachur, A., Osin, E., Davydov, D., Shutilov, K., \& Novokshonov, A. (2020). Assessing the Big Five personality traits using real-life static facial images. Scientific Reports, 10(1), 8487. https://doi.org/10.1038/s41598-020-65358-6 
Keltner, D., \& Haidt, J. (1999). Social functions of emotions at four levels of analysis. Cognition \& Emotion, 13(5), 505-521. https://doi.org/10.1080/026999399379168 Kittler, J., Huber, P., Feng, Z.-H., Hu, G., \& Christmas, W. (2016). 3D morphable face models and their applications. In F. J. Perales \& J. Kittler (Eds.), Articulated motion and deformable objects (pp. 185-206). Springer International Publishing. https://doi.org/10.1007/978-3-319-41778-3 19

Kosinski, M. (2021). Facial recognition technology can expose political orientation from naturalistic facial images. Scientific Reports, 11(1), 100. https://doi.org/10.1038/s41598-020-79310-1

Leary, M. R., \& Allen, A. B. (2011). Personality and persona: Personality processes in self-presentation. Journal of Personality, 79(6), 1191-1218. https://doi.org/10.1111/j.1467-6494.2010.00704.x

Leary, M. R., \& Kowalski, R. M. (1990). Impression management: A literature review and two-component model. Psychological Bulletin 107(1), 34-47.

Leary, M. R., Nezlek, J. B., Downs, D., Radford-Davenport, J., Martin, J., \& McMullen, A. (1994). Self-presentation in everyday interactions: Effects of target familiarity and gender composition. Journal of Personality and Social Psychology, 67(4), 664-673. https://doi.org/10.1037/0022-3514.67.4.664

Li, Y., Vishwamitra, N., Hu, H., Knijnenburg, B. P., \& Caine, K. (2017). Effectiveness and users' experience of face blurring as a privacy protection for sharing photos via online social networks. Proceedings of the Human Factors and Ergonomics Society Annual Meeting, 61(1), 803-807. https://doi.org/10.1177/1541931213601694 
Matz, S. C., Appel, R. E., \& Kosinski, M. (2020). Privacy in the age of psychological targeting. Current Opinion in Psychology, 31, 116-121. https://doi.org/10.1016/j.copsyc.2019.08.010

Matz, S. C., \& Harari, G. M. (2021). Personality-place transactions: Mapping the relationships between Big Five personality traits, states, and daily places. Journal of Personality and Social Psychology, 120(5), 1367-1385. https://doi.org/10.1037/pspp0000297

Matzner, T., Masur, P. K., Ochs, C., \& von Pape, T. (2016). Do-it-yourself data protection—empowerment or burden? In S. Gutwirth, R. Leenes, \& P. De Hert (Eds.), Data protection on the move: Current developments in ICT and privacy/data protection (pp. 277-305). Springer Netherlands. https://doi.org/10.1007/978-94-017-7376-8 11

Nicholls, M. E. R., Wolfgang, B. J., Clode, D., \& Lindell, A. K. (2002). The effect of left and right poses on the expression of facial emotion. Neuropsychologia, 40(10), 1662-1665. https://doi.org/10.1016/S0028-3932(02)00024-6

Noyes, E., \& Jenkins, R. (2017). Camera-to-subject distance affects face configuration and perceived identity. Cognition, 165, 97-104. https://doi.org/10.1016/j.cognition.2017.05.012

Olivola, C. Y., Funk, F., \& Todorov, A. (2014). Social attributions from faces bias human choices. Trends in Cognitive Sciences, 18(11), 566-570. https://doi.org/10.1016/j.tics.2014.09.007 
Olivola, C. Y., \& Todorov, A. (2010). Fooled by first impressions? Reexamining the diagnostic value of appearance-based inferences. Journal of Experimental Social Psychology, 46(2), 315-324. https://doi.org/10.1016/j.jesp.2009.12.002

Oosterhof, N. N., \& Todorov, A. (2008). The functional basis of face evaluation. Proceedings of the National Academy of Sciences, 105(32), 11087-11092. https://doi.org/10.1073/pnas.0805664105

Oosterhof, N., \& Todorov, A. (2009). Shared perceptual basis of emotional expressions and trustworthiness impressions from faces. Emotion, 9(1), 128-133.

\section{https://doi.org/10.1037/a0014520}

Ota, C., \& Nakano, T. (2021). Neural correlates of beauty retouching to enhance attractiveness of self-depictions in women. Social Neuroscience, 16(2), 121-133. https://doi.org/10.1080/17470919.2021.1873178

Parkhi, O. M., Vedaldi, A., \& Zisserman, A. (2015). Deep face recognition. Proceedings of the British Machine Vision Conference 2015, 41.1-41.12. https://doi.org/10.5244/C.29.41

Phillips, P. J. (2017). A cross benchmark assessment of a deep convolutional neural network for face recognition. 12th IEEE International Conference on Automatic Face Gesture Recognition (FG 2017), 705-710. https://doi.org/10.1109/FG.2017.89

Prasad, P., Pathak, R., Gunjan, V. K., \& Rao, H. V. (2020). Deep learning based representation for face recognition. Proceedings of the 2nd International Conference on Communications and Cyber Physical Engineering (pp. 419-424). https://doi.org/10.1007/978-981-13-8715-9 50 
Raschka, S. (2020). Model evaluation, model selection, and algorithm selection in machine learning. ArXiv:1811.12808 [Cs, Stat]. http://arxiv.org/abs/1811.12808

Reddy, V. (2000). Coyness in early infancy. Developmental Science, 3(2), 186-192. https://doi.org/10.1111/1467-7687.00112

Rezende, I. N. (2020). Facial recognition in police hands: Assessing the 'Clearview case' from a European perspective. New Journal of European Criminal Law, 11(3), 375-389. https://doi.org/10.1177/2032284420948161

Rothstein, M. A., \& Tovino, S. A. (2019). California takes the lead on data privacy law. Hastings Center Report, 49(5), 4-5. https://doi.org/10.1002/hast.1042

Rudd, N. A. (1996). Appearance and self-presentation research in gay consumer cultures: Issues and impact. Journal of Homosexuality, 31(1-2), 109-134. https://doi.org/10.1300/J082v31n01 07

Said, C. P., Sebe, N., \& Todorov, A. (2009). Structural resemblance to emotional expressions predicts evaluation of emotionally neutral faces. Emotion 9(2), 260264. https://doi.org/10.1037/A0014681

Sawilowsky, S. S. (2009). New effect size rules of thumb. Journal of Modern Applied Statistical Methods, 8(2), 597-599. https://doi.org/10.22237/jmasm/1257035100

Schau, H. J., \& Gilly, M. C. (2003). We are what we post? Self-presentation in personal web space. Journal of Consumer Research, 30(3), 385-404. https://doi.org/10.1086/378616

Schlenker, B. R. (2012). Self-presentation. In M. R. Leary \& J. P. Tangney (Eds.), Handbook of self and identity (2nd ed., pp. 542-570). The Guilford Press. 
Serengil, S. I., \& Ozpinar, A. (2020). LightFace: A hybrid deep face recognition framework. 2020 Innovations in Intelligent Systems and Applications Conference (ASYU), 1-5. https://doi.org/10.1109/ASYU50717.2020.9259802

Shan, S., Wenger, E., Zhang, J., Li, H., Zheng, H., \& Zhao, B. Y. (2020). Fawkes: Protecting privacy against unauthorized deep learning models. 1589-1604. https://www.usenix.org/conference/usenixsecurity20/presentation/shan

Shorten, C., \& Khoshgoftaar, T. (2019). A survey on image data augmentation for deep learning. Journal of Big Data, 6. https://doi.org/10.1186/s40537-019-0197-0

Stoker, J. I., Garretsen, H., \& Spreeuwers, L. J. (2016). The facial appearance of CEOs: faces signal selection but not performance. PLOS ONE, 11(7), e0159950. https://doi.org/10.1371/journal.pone.0159950

Susan, S., \& Kumar, A. (2021). The balancing trick: Optimized sampling of imbalanced datasets-A brief survey of the recent State of the Art. Engineering Reports, 3(4), e12298. https://doi.org/10.1002/eng2.12298

Taigman, Y., Yang, M., Ranzato, M., \& Wolf, L. (2015). Web-scale training for face identification. 2015 IEEE Conference on Computer Vision and Pattern Recognition (CVPR), 2746-2754. https://doi.org/10.1109/CVPR.2015.7298891

Todorov, A. (2008). Evaluating faces on trustworthiness. Annals of the New York Academy of Sciences, 1124(1), 208-224. https://doi.org/10.1196/annals.1440.012

Todorov, A., Baron, S. G., \& Oosterhof, N. N. (2008). Evaluating face trustworthiness: A model based approach. Social Cognitive and Affective Neuroscience, 3(2), 119127. https://doi.org/10.1093/scan/nsn009 
Todorov, A., Olivola, C. Y., Dotsch, R., \& Mende-Siedlecki, P. (2015). Social attributions from faces: Determinants, consequences, accuracy, and functional significance. Annual Review of Psychology, 66(1), 519-545. https://doi.org/10.1146/annurevpsych-113011-143831

Todorov, A., \& Porter, J. M. (2014). Misleading first impressions: Different for different facial images of the same person. Psychological Science, 25(7), 1404-1417. https://doi.org/10.1177/0956797614532474

Tong, S. T., Corriero, E. F., Wibowo, K. A., Makki, T. W., \& Slatcher, R. B. (2020). Selfpresentation and impressions of personality through text-based online dating profiles: A lens model analysis. New Media \& Society, 22(5), 875-895. https://doi.org/10.1177/1461444819872678

Torralba, A., Fergus, R., \& Freeman, W. T. (2008). 80 million tiny images: A large data set for nonparametric object and scene recognition. IEEE Transactions on Pattern Analysis and Machine Intelligence, 30(11), 1958-1970. https://doi.org/10.1109/TPAMI.2008.128

Tran, A. T., Hassner, T., Masi, I., Paz, E., Nirkin, Y., \& Medioni, G. (2018). Extreme 3D face reconstruction: Seeing through occlusions. 2018 IEEE/CVF Conference on Computer Vision and Pattern Recognition, 3935-3944. https://doi.org/10.1109/CVPR.2018.00414

Wang, D., Nair, K., Kouchaki, M., Zajac, E. J., \& Zhao, X. (2019). A case of evolutionary mismatch? Why facial width-to-height ratio may not predict behavioral tendencies. Psychological Science, 30(7), 1074-1081. https://doi.org/10.1177/0956797619849928 
Wang, Y., \& Kosinski, M. (2018). Deep neural networks are more accurate than humans at detecting sexual orientation from facial images. Journal of Personality and Social Psychology, 114(2), 246-257. https://doi.org/10.1037/pspa0000098

White, D., Sutherland, C. A. M., \& Burton, A. L. (2017). Choosing face: The curse of self in profile image selection. Cognitive Research: Principles and Implications, 2(1), 23. https://doi.org/10.1186/s41235-017-0058-3

Witkower, Z., \& Tracy, J. L. (2019). A facial-action imposter: How head tilt influences perceptions of dominance from a neutral face. Psychological Science, 30(6), 893-906. https://doi.org/10.1177/0956797619838762

Wolffhechel, K., Fagertun, J., Jacobsen, U. P., Majewski, W., Hemmingsen, A. S., Larsen, C. L., Lorentzen, S. K., \& Jarmer, H. (2014). Interpretation of appearance: The effect of facial features on first impressions and personality. PLOS ONE, 9(9), e107721. https://doi.org/10.1371/journal.pone.0107721

Wrzus, C., Wagner, G. G., \& Riediger, M. (2016). Personality-situation transactions from adolescence to old age. Journal of Personality and Social Psychology, 110(5), 782-799. https://doi.org/10.1037/pspp0000054

Wu, X., \& Zhang, X. (2016). Automated inference on criminality using face images. arXiv preprint arXiv:1611.04135, 4038-4052. http://arxiv.org/abs/1611.04135

Zeiler, M. D., \& Fergus, R. (2013). Visualizing and understanding convolutional networks. ArXiv:1311.2901 [Cs]. http://arxiv.org/abs/1311.2901 
Zhang, Y., Lu, Y., Nagahara, H., \& Taniguchi, R. (2014). Anonymous camera for privacy protection. 22nd International Conference on Pattern Recognition, 4170-4175. https://doi.org/10.1109/ICPR.2014.715 
Table S1.

Cronbach's Alpha Reliability of Within-Subject Facial Attributes for Study 1a

\begin{tabular}{lccccc}
\hline \multirow{2}{*}{ Attribute } & \multicolumn{3}{c}{ Women } & & \multicolumn{3}{c}{ Men } \\
\cline { 2 - 3 } \cline { 5 - 6 } Neutral & Reliability & $95 \% \mathrm{Cl}$ & Reliability & $95 \% \mathrm{Cl}$ \\
Happiness & 0.670 & {$[0.660,0.680]$} & & 0.681 & {$[0.667,0.694]$} \\
Anger & 0.729 & {$[0.721,0.737]$} & & 0.718 & {$[0.706,0.730]$} \\
Surprise & 0.141 & {$[0.115,0.167]$} & & 0.354 & {$[0.325,0.381]$} \\
Disgust & 0.370 & {$[0.350,0.389]$} & & 0.372 & {$[0.345,0.399]$} \\
Sadness & 0.239 & {$[0.216,0.262]$} & & 0.292 & {$[0.261,0.322]$} \\
Eyes & 0.228 & {$[0.204,0.251]$} & & 0.257 & {$[0.224,0.289]$} \\
Glasses & 0.372 & {$[0.353,0.391]$} & & 0.499 & {$[0.476,0.520]$} \\
Smiling & 0.794 & {$[0.787,0.800]$} & & 0.712 & {$[0.699,0.724]$} \\
Roll & 0.766 & {$[0.758,0.773]$} & & 0.726 & {$[0.714,0.738]$} \\
Yaw & 0.136 & {$[0.110,0.162]$} & & 0.225 & {$[0.191,0.258]$} \\
Pitch & 0.327 & {$[0.306,0.347]$} & & 0.327 & {$[0.297,0.355]$} \\
Average & 0.495 & {$[0.480,0.510]$} & & 0.545 & {$[0.525,0.565]$} \\
\hline
\end{tabular}


Table S2.

Sample Size by Cross-Validation Fold, Train-Test Partition and Number of Images Separately for Each Gender for Study $1 \mathrm{C}$

Panel A: Women

\begin{tabular}{|c|c|c|c|c|c|c|c|c|}
\hline \multirow{2}{*}{ Fold } & \multirow{2}{*}{ Split } & \multirow{2}{*}{ Participants } & \multirow{2}{*}{ Images } & \multicolumn{5}{|c|}{ Participants with: } \\
\hline & & & & 1 Image & 2 Images & 3 Images & 4 Images & $\geq 5$ Images \\
\hline \multirow{2}{*}{1} & Train & 9823 & 20520 & 4292 & 2405 & 1847 & 761 & 518 \\
\hline & Test & 517 & 1080 & 226 & 127 & 97 & 39 & 28 \\
\hline \multirow{2}{*}{2} & Train & 9823 & 20520 & 4292 & 2405 & 1847 & 761 & 518 \\
\hline & Test & 517 & 1080 & 226 & 127 & 97 & 39 & 28 \\
\hline \multirow{2}{*}{3} & Train & 9823 & 20520 & 4292 & 2405 & 1848 & 759 & 519 \\
\hline & Test & 517 & 1080 & 226 & 127 & 96 & 41 & 27 \\
\hline \multirow{2}{*}{4} & Train & 9823 & 20520 & 4292 & 2405 & 1848 & 759 & 519 \\
\hline & Test & 517 & 1080 & 226 & 127 & 96 & 41 & 27 \\
\hline \multirow{2}{*}{5} & Train & 9823 & 20520 & 4292 & 2405 & 1848 & 759 & 519 \\
\hline & Test & 517 & 1080 & 226 & 127 & 96 & 41 & 27 \\
\hline \multirow{2}{*}{6} & Train & 9823 & 20520 & 4292 & 2405 & 1848 & 759 & 519 \\
\hline & Test & 517 & 1080 & 226 & 127 & 96 & 41 & 27 \\
\hline \multirow{2}{*}{7} & Train & 9823 & 20520 & 4292 & 2405 & 1848 & 759 & 519 \\
\hline & Test & 517 & 1080 & 226 & 127 & 96 & 41 & 27 \\
\hline \multirow{2}{*}{8} & Train & 9823 & 20520 & 4292 & 2405 & 1848 & 759 & 519 \\
\hline & Test & 517 & 1080 & 226 & 127 & 96 & 41 & 27 \\
\hline \multirow{2}{*}{9} & Train & 9823 & 20520 & 4292 & 2406 & 1846 & 760 & 519 \\
\hline & Test & 517 & 1080 & 226 & 126 & 98 & 40 & 27 \\
\hline \multirow{2}{*}{10} & Train & 9823 & 20520 & 4292 & 2406 & 1846 & 760 & 519 \\
\hline & Test & 517 & 1080 & 226 & 126 & 98 & 40 & 27 \\
\hline \multirow{2}{*}{11} & Train & 9823 & 20520 & 4292 & 2406 & 1846 & 760 & 519 \\
\hline & Test & 517 & 1080 & 226 & 126 & 98 & 40 & 27 \\
\hline \multirow{2}{*}{12} & Train & 9823 & 20520 & 4292 & 2406 & 1846 & 760 & 519 \\
\hline & Test & 517 & 1080 & 226 & 126 & 98 & 40 & 27 \\
\hline \multirow{2}{*}{13} & Train & 9823 & 20520 & 4292 & 2406 & 1846 & 760 & 519 \\
\hline & Test & 517 & 1080 & 226 & 126 & 98 & 40 & 27 \\
\hline \multirow{2}{*}{14} & Train & 9823 & 20520 & 4292 & 2406 & 1846 & 760 & 519 \\
\hline & Test & 517 & 1080 & 226 & 126 & 98 & 40 & 27 \\
\hline \multirow{2}{*}{15} & Train & 9823 & 20520 & 4292 & 2406 & 1846 & 760 & 519 \\
\hline & Test & 517 & 1080 & 226 & 126 & 98 & 40 & 27 \\
\hline \multirow{2}{*}{16} & Train & 9823 & 20520 & 4292 & 2406 & 1846 & 760 & 519 \\
\hline & Test & 517 & 1080 & 226 & 126 & 98 & 40 & 27 \\
\hline \multirow{2}{*}{17} & Train & 9823 & 20520 & 4292 & 2406 & 1846 & 761 & 518 \\
\hline & Test & 517 & 1080 & 226 & 126 & 98 & 39 & 28 \\
\hline \multirow{2}{*}{18} & Train & 9823 & 20520 & 4292 & 2406 & 1846 & 761 & 518 \\
\hline & Test & 517 & 1080 & 226 & 126 & 98 & 39 & 28 \\
\hline 19 & Train & 9823 & 20520 & 4293 & 2404 & 1847 & 761 & 518 \\
\hline 19 & Test & 517 & 1080 & 225 & 128 & 97 & 39 & 28 \\
\hline & Train & 9823 & 20520 & 4293 & 2404 & 1847 & 761 & 518 \\
\hline 20 & Test & 517 & 1080 & 225 & 128 & 97 & 39 & 28 \\
\hline & & 10340 & 21600 & 4518 & 2532 & 1944 & 800 & 546 \\
\hline
\end{tabular}


Panel B: Men

\begin{tabular}{|c|c|c|c|c|c|c|c|c|}
\hline \multirow{2}{*}{ Fold } & \multirow{2}{*}{ Split } & \multirow{2}{*}{ Participants } & \multirow{2}{*}{ Images } & \multicolumn{5}{|c|}{ Participants with: } \\
\hline & & & & 1 Image & 2 Images & 3 Images & 4 Images & $\geq 5$ Images \\
\hline \multirow{2}{*}{1} & Train & 4867 & 9653 & 2258 & 1218 & 863 & 336 & 192 \\
\hline & Test & 257 & 509 & 120 & 64 & 45 & 18 & 10 \\
\hline \multirow{2}{*}{2} & Train & 4867 & 9653 & 2258 & 1218 & 863 & 336 & 192 \\
\hline & Test & 257 & 509 & 120 & 64 & 45 & 18 & 10 \\
\hline \multirow{2}{*}{3} & Train & 4868 & 9654 & 2259 & 1218 & 863 & 336 & 192 \\
\hline & Test & 256 & 508 & 119 & 64 & 45 & 18 & 10 \\
\hline \multirow{2}{*}{4} & Train & 4868 & 9654 & 2259 & 1218 & 863 & 336 & 192 \\
\hline & Test & 256 & 508 & 119 & 64 & 45 & 18 & 10 \\
\hline \multirow{2}{*}{5} & Train & 4868 & 9654 & 2259 & 1218 & 863 & 336 & 192 \\
\hline & Test & 256 & 508 & 119 & 64 & 45 & 18 & 10 \\
\hline \multirow{2}{*}{6} & Train & 4868 & 9654 & 2259 & 1218 & 863 & 336 & 192 \\
\hline & Test & 256 & 508 & 119 & 64 & 45 & 18 & 10 \\
\hline \multirow{2}{*}{7} & Train & 4868 & 9654 & 2259 & 1218 & 863 & 336 & 192 \\
\hline & Test & 256 & 508 & 119 & 64 & 45 & 18 & 10 \\
\hline \multirow{2}{*}{8} & Train & 4868 & 9654 & 2259 & 1218 & 863 & 336 & 192 \\
\hline & Test & 256 & 508 & 119 & 64 & 45 & 18 & 10 \\
\hline \multirow{2}{*}{9} & Train & 4868 & 9654 & 2259 & 1219 & 861 & 337 & 192 \\
\hline & Test & 256 & 508 & 119 & 63 & 47 & 17 & 10 \\
\hline \multirow{2}{*}{10} & Train & 4868 & 9654 & 2259 & 1219 & 861 & 337 & 192 \\
\hline & Test & 256 & 508 & 119 & 63 & 47 & 17 & 10 \\
\hline \multirow{2}{*}{11} & Train & 4868 & 9654 & 2259 & 1219 & 861 & 338 & 191 \\
\hline & Test & 256 & 508 & 119 & 63 & 47 & 16 & 11 \\
\hline \multirow{2}{*}{12} & Train & 4868 & 9654 & 2259 & 1219 & 861 & 338 & 191 \\
\hline & Test & 256 & 508 & 119 & 63 & 47 & 16 & 11 \\
\hline \multirow{2}{*}{13} & Train & 4868 & 9654 & 2260 & 1217 & 863 & 336 & 192 \\
\hline & Test & 256 & 508 & 118 & 65 & 45 & 18 & 10 \\
\hline \multirow{2}{*}{14} & Train & 4868 & 9654 & 2260 & 1217 & 863 & 336 & 192 \\
\hline & Test & 256 & 508 & 118 & 65 & 45 & 18 & 10 \\
\hline \multirow{2}{*}{15} & Train & 4868 & 9654 & 2260 & 1217 & 863 & 336 & 192 \\
\hline & Test & 256 & 508 & 118 & 65 & 45 & 18 & 10 \\
\hline \multirow{2}{*}{16} & Train & 4868 & 9654 & 2260 & 1217 & 863 & 336 & 192 \\
\hline & Test & 256 & 508 & 118 & 65 & 45 & 18 & 10 \\
\hline \multirow{2}{*}{17} & Train & 4868 & 9654 & 2260 & 1217 & 863 & 336 & 192 \\
\hline & Test & 256 & 508 & 118 & 65 & 45 & 18 & 10 \\
\hline \multirow{2}{*}{18} & Train & 4868 & 9654 & 2260 & 1217 & 863 & 336 & 192 \\
\hline & Test & 256 & 508 & 118 & 65 & 45 & 18 & 10 \\
\hline 19 & Train & 4867 & 9654 & 2258 & 1218 & 863 & 336 & 192 \\
\hline 19 & Test & 257 & 508 & 120 & 64 & 45 & 18 & 10 \\
\hline & Train & 4867 & 9654 & 2258 & 1218 & 863 & 336 & 192 \\
\hline 20 & Test & 257 & 508 & 120 & 64 & 45 & 18 & 10 \\
\hline & & 5124 & 10162 & 2378 & 1282 & 908 & 354 & 202 \\
\hline
\end{tabular}


Table S3.

Average AUC Results by Different Degrees of Masking Compared to AUC of Random (Fully Masked Images) for Study 1c

\begin{tabular}{|c|c|c|c|c|c|c|c|c|}
\hline \multirow[b]{2}{*}{ Degree } & \multicolumn{4}{|c|}{ Women $(\mathrm{N}=10,340)$} & \multicolumn{4}{|c|}{$\operatorname{Men}(\mathrm{N}=5,124)$} \\
\hline & AUC & $95 \% \mathrm{Cl}$ & $p$ & $d$ & AUC & $95 \% \mathrm{Cl}$ & $p$ & $d$ \\
\hline Baseline & .702 & {$[.692, .712]$} & $<.001$ & 4.002 & .662 & {$[.647, .677]$} & $<.001$ & 2.643 \\
\hline $3 \%$ & .695 & {$[.685, .705]$} & $<.001$ & 3.856 & .660 & {$[.645, .674]$} & $<.001$ & 2.600 \\
\hline $6 \%$ & .680 & {$[.670, .690]$} & $<.001$ & 3.528 & .661 & {$[.646, .675]$} & $<.001$ & 2.616 \\
\hline $10 \%$ & .665 & {$[.654, .675]$} & $<.001$ & 3.201 & .652 & {$[.637, .667]$} & $<.001$ & 2.461 \\
\hline $13 \%$ & .657 & {$[.646, .667]$} & $<.001$ & 3.035 & .644 & {$[.629, .659]$} & $<.001$ & 2.332 \\
\hline $16 \%$ & .652 & {$[.641, .662]$} & $<.001$ & 2.935 & .638 & {$[.623, .653]$} & $<.001$ & 2.225 \\
\hline $20 \%$ & .647 & {$[.637, .658]$} & $<.001$ & 2.841 & .633 & {$[.618, .648]$} & $<.001$ & 2.146 \\
\hline $23 \%$ & .645 & {$[.635, .656]$} & $<.001$ & 2.800 & .628 & {$[.613, .644]$} & $<.001$ & 2.064 \\
\hline $26 \%$ & .642 & {$[.631, .652]$} & $<.001$ & 2.731 & .623 & {$[.608, .638]$} & $<.001$ & 1.975 \\
\hline $30 \%$ & .639 & {$[.629, .650]$} & $<.001$ & 2.684 & .622 & {$[.607, .637]$} & $<.001$ & 1.956 \\
\hline $33 \%$ & .636 & {$[.625, .646]$} & $<.001$ & 2.606 & .619 & {$[.604, .634]$} & $<.001$ & 1.905 \\
\hline $36 \%$ & .634 & {$[.624, .645]$} & $<.001$ & 2.577 & .615 & {$[.600, .630]$} & $<.001$ & 1.842 \\
\hline $40 \%$ & .631 & {$[.620, .641]$} & $<.001$ & 2.510 & .605 & {$[.589, .620]$} & $<.001$ & 1.673 \\
\hline $43 \%$ & .629 & {$[.619, .640]$} & $<.001$ & 2.477 & .606 & {$[.590, .621]$} & $<.001$ & 1.685 \\
\hline $46 \%$ & .624 & {$[.614, .635]$} & $<.001$ & 2.382 & .607 & {$[.591, .622]$} & $<.001$ & 1.701 \\
\hline $50 \%$ & .620 & {$[.609, .631]$} & $<.001$ & 2.295 & .602 & {$[.587, .618]$} & $<.001$ & 1.631 \\
\hline $53 \%$ & .615 & {$[.604, .626]$} & $<.001$ & 2.192 & .595 & {$[.580, .611]$} & $<.001$ & 1.518 \\
\hline $56 \%$ & .612 & {$[.601, .623]$} & $<.001$ & 2.134 & .596 & {$[.581, .612]$} & $<.001$ & 1.533 \\
\hline $60 \%$ & .605 & {$[.594, .616]$} & $<.001$ & 2.000 & .600 & {$[.585, .616]$} & $<.001$ & 1.596 \\
\hline $63 \%$ & .605 & {$[.594, .616]$} & $<.001$ & 1.996 & .593 & {$[.578, .609]$} & $<.001$ & 1.487 \\
\hline $66 \%$ & .599 & {$[.588, .610]$} & $<.001$ & 1.885 & .591 & {$[.576, .607]$} & $<.001$ & 1.449 \\
\hline $70 \%$ & .592 & {$[.581, .603]$} & $<.001$ & 1.742 & .589 & {$[.574, .605]$} & $<.001$ & 1.419 \\
\hline $73 \%$ & .586 & {$[.575, .597]$} & $<.001$ & 1.632 & .589 & {$[.573, .604]$} & $<.001$ & 1.408 \\
\hline $76 \%$ & .582 & {$[.571, .593]$} & $<.001$ & 1.557 & .583 & {$[.568, .599]$} & $<.001$ & 1.324 \\
\hline $80 \%$ & .575 & {$[.564, .586]$} & $<.001$ & 1.420 & .580 & {$[.564, .595]$} & $<.001$ & 1.267 \\
\hline $83 \%$ & .567 & {$[.556, .578]$} & $<.001$ & 1.260 & .579 & {$[.563, .594]$} & $<.001$ & 1.246 \\
\hline $86 \%$ & .557 & {$[.546, .568]$} & $<.001$ & 1.082 & .578 & {$[.563, .594]$} & $<.001$ & 1.241 \\
\hline $90 \%$ & .554 & {$[.543, .565]$} & $<.001$ & 1.021 & .576 & {$[.560, .591]$} & $<.001$ & 1.200 \\
\hline $93 \%$ & .552 & {$[.541, .563]$} & $<.001$ & 0.975 & .575 & {$[.559, .590]$} & $<.001$ & 1.187 \\
\hline $96 \%$ & .540 & {$[.529, .551]$} & $<.001$ & 0.760 & .568 & {$[.552, .583]$} & $<.001$ & 1.072 \\
\hline
\end{tabular}

Note. Average AUC was generated by the following steps. First, averaging the predictions across facial images for the same person. Second, comparing the averaged prediction to the observed value to generate the average AUC. 
Table S4.

AUC Results Using One Image by Different Degrees of Masking Compared to AUC of Random (Fully Masked Images) for Study $1 \mathrm{c}$

\begin{tabular}{|c|c|c|c|c|c|c|c|c|}
\hline \multirow[b]{2}{*}{ Degree } & \multicolumn{4}{|c|}{ Women $(\mathrm{N}=10,340)$} & \multicolumn{4}{|c|}{$\operatorname{Men}(\mathrm{N}=5,124)$} \\
\hline & AUC & $95 \% \mathrm{Cl}$ & $p$ & $d$ & AUC & $95 \% \mathrm{Cl}$ & $p$ & $d$ \\
\hline Baseline & .687 & {$[.677, .697]$} & $<.001$ & 3.679 & .644 & {$[.630, .659]$} & $<.001$ & 2.340 \\
\hline $3 \%$ & .679 & {$[.669, .689]$} & $<.001$ & 3.514 & .644 & {$[.629, .659]$} & $<.001$ & 2.323 \\
\hline $6 \%$ & .663 & {$[.653, .673]$} & $<.001$ & 3.169 & .639 & {$[.624, .654]$} & $<.001$ & 2.244 \\
\hline $10 \%$ & .648 & {$[.638, .659]$} & $<.001$ & 2.860 & .632 & {$[.617, .647]$} & $<.001$ & 2.121 \\
\hline $13 \%$ & .640 & {$[.629, .650]$} & $<.001$ & 2.693 & .628 & {$[.613, .643]$} & $<.001$ & 2.056 \\
\hline $16 \%$ & .636 & {$[.626, .647]$} & $<.001$ & 2.624 & .625 & {$[.610, .640]$} & $<.001$ & 2.008 \\
\hline $20 \%$ & .632 & {$[.622, .643]$} & $<.001$ & 2.544 & .621 & {$[.606, .636]$} & $<.001$ & 1.939 \\
\hline $23 \%$ & .631 & {$[.620, .642]$} & $<.001$ & 2.513 & .614 & {$[.599, .630]$} & $<.001$ & 1.831 \\
\hline $26 \%$ & .628 & {$[.618, .639]$} & $<.001$ & 2.460 & 610 & {$[.595, .626]$} & $<.001$ & 1.765 \\
\hline $30 \%$ & .626 & {$[.615, .636]$} & $<.001$ & 2.409 & .610 & {$[.594, .625]$} & $<.001$ & 1.751 \\
\hline $33 \%$ & .624 & {$[.613, .635]$} & $<.001$ & 2.376 & .605 & {$[.590, .620]$} & $<.001$ & 1.678 \\
\hline $36 \%$ & .623 & {$[.612, .633]$} & $<.001$ & 2.344 & .603 & {$[.588, .618]$} & $<.001$ & 1.643 \\
\hline $40 \%$ & .620 & {$[.609, .630]$} & $<.001$ & 2.285 & .592 & {$[.577, .608]$} & $<.001$ & 1.473 \\
\hline $43 \%$ & .618 & {$[.607, .628]$} & $<.001$ & 2.246 & .592 & {$[.576, .607]$} & $<.001$ & 1.459 \\
\hline $46 \%$ & .612 & {$[.601, .623]$} & $<.001$ & 2.141 & .592 & {$[.577, .608]$} & $<.001$ & 1.472 \\
\hline $50 \%$ & .608 & {$[.597, .618]$} & $<.001$ & 2.050 & .589 & {$[.574, .605]$} & $<.001$ & 1.419 \\
\hline $53 \%$ & .604 & {$[.593, .614]$} & $<.001$ & 1.972 & .582 & {$[.567, .598]$} & $<.001$ & 1.305 \\
\hline $56 \%$ & .601 & {$[.590, .611]$} & $<.001$ & 1.912 & .582 & {$[.566, .597]$} & $<.001$ & 1.302 \\
\hline $60 \%$ & .594 & {$[.583, .605]$} & $<.001$ & 1.778 & .585 & {$[.570, .601]$} & $<.001$ & 1.353 \\
\hline $63 \%$ & .593 & {$[.582, .604]$} & $<.001$ & 1.758 & .578 & {$[.562, .593]$} & $<.001$ & 1.236 \\
\hline $66 \%$ & .587 & {$[.576, .598]$} & $<.001$ & 1.645 & .576 & {$[.560, .592]$} & $<.001$ & 1.206 \\
\hline $70 \%$ & .580 & {$[.569, .591]$} & $<.001$ & 1.509 & .574 & {$[.558, .589]$} & $<.001$ & 1.166 \\
\hline $73 \%$ & .574 & {$[.564, .585]$} & $<.001$ & 1.408 & .573 & {$[.557, .588]$} & $<.001$ & 1.150 \\
\hline $76 \%$ & .567 & {$[.556, .578]$} & $<.001$ & 1.271 & .567 & {$[.552, .583]$} & $<.001$ & 1.069 \\
\hline $80 \%$ & .563 & {$[.552, .574]$} & $<.001$ & 1.195 & .564 & {$[.548, .580]$} & $<.001$ & 1.013 \\
\hline $83 \%$ & .555 & {$[.544, .566]$} & $<.001$ & 1.032 & .564 & {$[.548, .580]$} & $<.001$ & 1.014 \\
\hline $86 \%$ & .546 & {$[.535, .557]$} & $<.001$ & 0.862 & .564 & {$[.549, .580]$} & $<.001$ & 1.017 \\
\hline $90 \%$ & .545 & {$[.534, .556]$} & $<.001$ & 0.851 & .563 & {$[.547, .579]$} & $<.001$ & 0.998 \\
\hline $93 \%$ & .543 & {$[.532, .555]$} & $<.001$ & 0.818 & .561 & {$[.546, .577]$} & $<.001$ & 0.970 \\
\hline $96 \%$ & .534 & {$[.523, .545]$} & $<.001$ & 0.643 & .556 & {$[.540, .572]$} & $<.001$ & 0.884 \\
\hline
\end{tabular}


Table S5.

Average Accuracy Results by Different Degrees of Masking for Study $1 \mathrm{c}$

\begin{tabular}{|c|c|c|c|c|c|c|c|c|}
\hline \multirow[b]{2}{*}{ Degree } & \multicolumn{4}{|c|}{ Women } & \multicolumn{4}{|c|}{ Men } \\
\hline & Accuracy & Precision & Recall & F1 & Accuracy & Precision & Recall & F1 \\
\hline Baseline & $64.83 \%$ & $64.72 \%$ & $65.18 \%$ & $64.95 \%$ & $61.10 \%$ & $61.21 \%$ & $60.66 \%$ & $60.93 \%$ \\
\hline $3 \%$ & $64.49 \%$ & $64.57 \%$ & $64.20 \%$ & $64.38 \%$ & $61.55 \%$ & $61.91 \%$ & $60.07 \%$ & $60.97 \%$ \\
\hline $6 \%$ & $63.05 \%$ & $63.32 \%$ & $62.03 \%$ & $62.67 \%$ & $61.94 \%$ & $62.50 \%$ & $59.72 \%$ & $61.08 \%$ \\
\hline $10 \%$ & $61.97 \%$ & $62.20 \%$ & $61.04 \%$ & $61.62 \%$ & $61.49 \%$ & $62.35 \%$ & $58.04 \%$ & $60.12 \%$ \\
\hline $13 \%$ & $61.40 \%$ & $61.41 \%$ & $61.35 \%$ & $61.38 \%$ & $60.85 \%$ & $61.87 \%$ & $56.56 \%$ & $59.09 \%$ \\
\hline $16 \%$ & $60.84 \%$ & $60.92 \%$ & $60.50 \%$ & $60.71 \%$ & $60.13 \%$ & $61.06 \%$ & $55.93 \%$ & $58.38 \%$ \\
\hline $20 \%$ & $60.33 \%$ & $60.39 \%$ & $60.02 \%$ & $60.21 \%$ & $60.21 \%$ & $60.91 \%$ & $56.99 \%$ & $58.88 \%$ \\
\hline $23 \%$ & $60.09 \%$ & $60.12 \%$ & $59.92 \%$ & $60.02 \%$ & $59.95 \%$ & $60.88 \%$ & $55.70 \%$ & $58.17 \%$ \\
\hline $26 \%$ & $60.13 \%$ & $60.18 \%$ & $59.85 \%$ & $60.01 \%$ & $59.93 \%$ & $61.03 \%$ & $54.96 \%$ & $57.84 \%$ \\
\hline $30 \%$ & $60.05 \%$ & $60.03 \%$ & $60.14 \%$ & $60.08 \%$ & $59.62 \%$ & $60.41 \%$ & $55.85 \%$ & $58.04 \%$ \\
\hline $33 \%$ & $59.73 \%$ & $59.73 \%$ & $59.71 \%$ & $59.72 \%$ & $58.65 \%$ & $59.54 \%$ & $53.98 \%$ & $56.62 \%$ \\
\hline $36 \%$ & $59.97 \%$ & $59.87 \%$ & $60.46 \%$ & $60.17 \%$ & $57.77 \%$ & $58.36 \%$ & $54.22 \%$ & $56.21 \%$ \\
\hline $40 \%$ & $59.56 \%$ & $59.38 \%$ & $60.56 \%$ & $59.96 \%$ & $57.16 \%$ & $57.55 \%$ & $54.61 \%$ & $56.04 \%$ \\
\hline $43 \%$ & $59.26 \%$ & $59.11 \%$ & $60.06 \%$ & $59.58 \%$ & $57.42 \%$ & $57.71 \%$ & $55.50 \%$ & $56.59 \%$ \\
\hline $46 \%$ & $58.95 \%$ & $58.75 \%$ & $60.06 \%$ & $59.40 \%$ & $57.90 \%$ & $58.16 \%$ & $56.32 \%$ & $57.23 \%$ \\
\hline $50 \%$ & $58.74 \%$ & $58.52 \%$ & $60.08 \%$ & $59.29 \%$ & $57.81 \%$ & $58.01 \%$ & $56.52 \%$ & $57.26 \%$ \\
\hline $53 \%$ & $58.11 \%$ & $57.91 \%$ & $59.40 \%$ & $58.65 \%$ & $56.95 \%$ & $57.03 \%$ & $56.36 \%$ & $56.69 \%$ \\
\hline $56 \%$ & $57.68 \%$ & $57.40 \%$ & $59.59 \%$ & $58.47 \%$ & $56.81 \%$ & $56.88 \%$ & $56.28 \%$ & $56.58 \%$ \\
\hline $60 \%$ & $57.53 \%$ & $57.30 \%$ & $59.17 \%$ & $58.22 \%$ & $57.61 \%$ & $57.67 \%$ & $57.26 \%$ & $57.46 \%$ \\
\hline $63 \%$ & $57.33 \%$ & $57.13 \%$ & $58.72 \%$ & $57.92 \%$ & $56.79 \%$ & $56.77 \%$ & $56.99 \%$ & $56.88 \%$ \\
\hline $66 \%$ & $57.05 \%$ & $56.88 \%$ & $58.30 \%$ & $57.58 \%$ & $56.42 \%$ & $56.29 \%$ & $57.46 \%$ & $56.87 \%$ \\
\hline $70 \%$ & $56.76 \%$ & $56.59 \%$ & $58.05 \%$ & $57.31 \%$ & $57.06 \%$ & $56.80 \%$ & $59.02 \%$ & $57.89 \%$ \\
\hline $73 \%$ & $56.19 \%$ & $56.15 \%$ & $56.48 \%$ & $56.32 \%$ & $56.32 \%$ & $56.12 \%$ & $58.00 \%$ & $57.04 \%$ \\
\hline $76 \%$ & $55.94 \%$ & $55.89 \%$ & $56.32 \%$ & $56.11 \%$ & $55.58 \%$ & $55.37 \%$ & $57.53 \%$ & $56.43 \%$ \\
\hline $80 \%$ & $55.08 \%$ & $55.10 \%$ & $54.89 \%$ & $54.99 \%$ & $55.68 \%$ & $55.42 \%$ & $58.08 \%$ & $56.72 \%$ \\
\hline $83 \%$ & $54.57 \%$ & $54.64 \%$ & $53.83 \%$ & $54.23 \%$ & $55.48 \%$ & $55.24 \%$ & $57.77 \%$ & $56.48 \%$ \\
\hline $86 \%$ & $54.44 \%$ & $54.64 \%$ & $52.28 \%$ & $53.43 \%$ & $55.21 \%$ & $55.03 \%$ & $57.03 \%$ & $56.01 \%$ \\
\hline $90 \%$ & $54.26 \%$ & $54.43 \%$ & $52.32 \%$ & $53.35 \%$ & $55.17 \%$ & $55.01 \%$ & $56.79 \%$ & $55.89 \%$ \\
\hline $93 \%$ & $53.86 \%$ & $53.92 \%$ & $53.09 \%$ & $53.50 \%$ & $55.23 \%$ & $55.02 \%$ & $57.34 \%$ & $56.15 \%$ \\
\hline $96 \%$ & $53.00 \%$ & $53.09 \%$ & $51.45 \%$ & $52.26 \%$ & $54.25 \%$ & $54.06 \%$ & $56.67 \%$ & $55.34 \%$ \\
\hline Random & $50.00 \%$ & $50.00 \%$ & $100.00 \%$ & $66.67 \%$ & $50.00 \%$ & $50.00 \%$ & $100.00 \%$ & $66.67 \%$ \\
\hline
\end{tabular}

Note. Average accuracy was generated by the following steps. First, averaging the predictions across facial images for the same person. Second, comparing the averaged prediction to the observed value to generate the average accuracy. 
Table S6.

Accuracy Results for One Image Only by Different Degrees of Masking for Study $1 \mathrm{c}$

\begin{tabular}{|c|c|c|c|c|c|c|c|c|}
\hline \multirow[b]{2}{*}{ Degree } & \multicolumn{4}{|c|}{ Women } & \multicolumn{4}{|c|}{ Men } \\
\hline & Accuracy & Precision & Recall & F1 & Accuracy & Precision & Recall & F1 \\
\hline 0 & $63.64 \%$ & $63.39 \%$ & $64.55 \%$ & $63.96 \%$ & $59.37 \%$ & $59.44 \%$ & $58.98 \%$ & $59.21 \%$ \\
\hline 1 & $62.73 \%$ & $62.68 \%$ & $62.92 \%$ & $62.80 \%$ & $59.84 \%$ & $60.29 \%$ & $57.65 \%$ & $58.94 \%$ \\
\hline 2 & $61.71 \%$ & $61.77 \%$ & $61.45 \%$ & $61.61 \%$ & $59.31 \%$ & $59.84 \%$ & $56.64 \%$ & $58.19 \%$ \\
\hline 3 & $60.57 \%$ & $60.68 \%$ & $60.08 \%$ & $60.38 \%$ & $60.03 \%$ & $60.79 \%$ & $56.52 \%$ & $58.58 \%$ \\
\hline 4 & $60.04 \%$ & $60.02 \%$ & $60.12 \%$ & $60.07 \%$ & $59.15 \%$ & $59.95 \%$ & $55.15 \%$ & $57.45 \%$ \\
\hline 5 & $59.60 \%$ & $59.53 \%$ & $59.98 \%$ & $59.76 \%$ & $58.63 \%$ & $59.44 \%$ & $54.29 \%$ & $56.75 \%$ \\
\hline 6 & $59.54 \%$ & $59.51 \%$ & $59.69 \%$ & $59.60 \%$ & $59.17 \%$ & $59.80 \%$ & $55.97 \%$ & $57.82 \%$ \\
\hline 7 & $59.39 \%$ & $59.43 \%$ & $59.17 \%$ & $59.30 \%$ & $58.39 \%$ & $59.09 \%$ & $54.53 \%$ & $56.72 \%$ \\
\hline 8 & $59.18 \%$ & $59.21 \%$ & $59.01 \%$ & $59.11 \%$ & $58.80 \%$ & $59.67 \%$ & $54.29 \%$ & $56.86 \%$ \\
\hline 9 & $59.29 \%$ & $59.25 \%$ & $59.54 \%$ & $59.39 \%$ & $57.85 \%$ & $58.49 \%$ & $54.06 \%$ & $56.19 \%$ \\
\hline 10 & $58.81 \%$ & $58.76 \%$ & $59.11 \%$ & $58.93 \%$ & $57.20 \%$ & $58.03 \%$ & $52.03 \%$ & $54.87 \%$ \\
\hline 11 & $58.86 \%$ & $58.78 \%$ & $59.28 \%$ & $59.03 \%$ & $57.01 \%$ & $57.62 \%$ & $53.01 \%$ & $55.21 \%$ \\
\hline 12 & $58.53 \%$ & $58.36 \%$ & $59.56 \%$ & $58.95 \%$ & $56.05 \%$ & $56.38 \%$ & $53.43 \%$ & $54.87 \%$ \\
\hline 13 & $58.39 \%$ & $58.24 \%$ & $59.32 \%$ & $58.78 \%$ & $56.36 \%$ & $56.64 \%$ & $54.29 \%$ & $55.44 \%$ \\
\hline 14 & $58.03 \%$ & $57.83 \%$ & $59.28 \%$ & $58.55 \%$ & $56.26 \%$ & $56.42 \%$ & $55.04 \%$ & $55.72 \%$ \\
\hline 15 & $57.71 \%$ & $57.46 \%$ & $59.38 \%$ & $58.40 \%$ & $55.84 \%$ & $56.02 \%$ & $54.29 \%$ & $55.14 \%$ \\
\hline 16 & $57.43 \%$ & $57.22 \%$ & $58.84 \%$ & $58.02 \%$ & $55.58 \%$ & $55.64 \%$ & $55.04 \%$ & $55.34 \%$ \\
\hline 17 & $57.07 \%$ & $56.82 \%$ & $58.88 \%$ & $57.83 \%$ & $55.39 \%$ & $55.49 \%$ & $54.45 \%$ & $54.96 \%$ \\
\hline 18 & $56.67 \%$ & $56.42 \%$ & $58.63 \%$ & $57.50 \%$ & $56.30 \%$ & $56.35 \%$ & $55.97 \%$ & $56.16 \%$ \\
\hline 19 & $56.35 \%$ & $56.15 \%$ & $58.05 \%$ & $57.08 \%$ & $55.25 \%$ & $55.18 \%$ & $55.93 \%$ & $55.55 \%$ \\
\hline 20 & $55.80 \%$ & $55.68 \%$ & $56.87 \%$ & $56.27 \%$ & $55.00 \%$ & $54.92 \%$ & $55.74 \%$ & $55.33 \%$ \\
\hline 21 & $55.67 \%$ & $55.49 \%$ & $57.27 \%$ & $56.37 \%$ & $55.41 \%$ & $55.22 \%$ & $57.18 \%$ & $56.18 \%$ \\
\hline 22 & $55.21 \%$ & $55.17 \%$ & $55.59 \%$ & $55.38 \%$ & $55.21 \%$ & $55.10 \%$ & $56.32 \%$ & $55.70 \%$ \\
\hline 23 & $54.42 \%$ & $54.35 \%$ & $55.20 \%$ & $54.77 \%$ & $54.02 \%$ & $53.82 \%$ & $56.60 \%$ & $55.18 \%$ \\
\hline 24 & $54.04 \%$ & $54.01 \%$ & $54.39 \%$ & $54.20 \%$ & $53.96 \%$ & $53.77 \%$ & $56.44 \%$ & $55.08 \%$ \\
\hline 25 & $53.59 \%$ & $53.66 \%$ & $52.65 \%$ & $53.15 \%$ & $53.98 \%$ & $53.75 \%$ & $57.06 \%$ & $55.36 \%$ \\
\hline 26 & $53.22 \%$ & $53.37 \%$ & $51.04 \%$ & $52.18 \%$ & $54.47 \%$ & $54.25 \%$ & $57.03 \%$ & $55.60 \%$ \\
\hline 27 & $53.15 \%$ & $53.30 \%$ & $50.95 \%$ & $52.10 \%$ & $54.31 \%$ & $54.12 \%$ & $56.67 \%$ & $55.37 \%$ \\
\hline 28 & $53.20 \%$ & $53.25 \%$ & $52.40 \%$ & $52.82 \%$ & $54.63 \%$ & $54.40 \%$ & $57.14 \%$ & $55.74 \%$ \\
\hline 29 & $52.38 \%$ & $52.46 \%$ & $50.74 \%$ & $51.58 \%$ & $53.69 \%$ & $53.46 \%$ & $57.03 \%$ & $55.18 \%$ \\
\hline 30 & $50.00 \%$ & $50.00 \%$ & $100.00 \%$ & $66.67 \%$ & $50.00 \%$ & $50.00 \%$ & $100.00 \%$ & $66.67 \%$ \\
\hline
\end{tabular}


Table S7.

Statistical Tests of Brightness Differences by Gender and Facial Regions for Study $2 a$

Panel A: Brightness Difference in Image Background

\begin{tabular}{|c|c|c|c|c|c|c|c|c|}
\hline & \multicolumn{8}{|c|}{ Women $(\mathrm{N}=10,340)$} \\
\hline & \multicolumn{2}{|c|}{ Heterosexual } & \multicolumn{2}{|c|}{ Lesbian } & \multicolumn{4}{|c|}{ Significance Test } \\
\hline & Mean & $95 \% \mathrm{Cl}$ & Mean & $95 \% \mathrm{Cl}$ & $t$ & $95 \% \mathrm{Cl}$ & $p$ & $d$ \\
\hline Red & 0.400 & {$[0.40,0.40]$} & 0.387 & {$[0.38,0.39]$} & -5.328 & {$[-0.02,-0.01$} & $<.001$ & 0.072 \\
\hline Green & 0.352 & {$[0.35,0.36]$} & 0.342 & {$[0.34,0.34]$} & -4.998 & {$[-0.01,-0.01$} & $<.001$ & 0.068 \\
\hline Blue & 0.326 & {$[0.32,0.33]$} & 0.315 & {$[0.31,0.32]$} & -5.137 & {$[-0.01,-0.01$} & $<.001$ & 0.070 \\
\hline \multirow[t]{4}{*}{ Average } & 0.359 & {$[0.36,0.36]$} & 0.348 & {$[0.35,0.35]$} & -5.329 & {$[-0.02,-0.01$} & $<.001$ & 0.073 \\
\hline & \multicolumn{8}{|c|}{ Men $(N=5,124)$} \\
\hline & \multicolumn{2}{|c|}{ Heterosexual } & \multicolumn{2}{|r|}{ Gay } & \multicolumn{4}{|c|}{ Significance Test } \\
\hline & Mean & $95 \% \mathrm{Cl}$ & Mean & $95 \% \mathrm{Cl}$ & $t$ & $95 \% \mathrm{Cl}$ & $p$ & $d$ \\
\hline Red & 0.423 & {$[0.42,0.43]$} & 0.443 & {$[0.44,0.45]$} & 5.532 & {$[0.01,0.03]$} & $<.001$ & 0.110 \\
\hline Green & 0.394 & {$[0.39,0.40]$} & 0.413 & {$[0.41,0.42]$} & 5.312 & {$[0.01,0.03]$} & $<.001$ & 0.105 \\
\hline Blue & 0.373 & {$[0.37,0.38]$} & 0.389 & {$[0.38,0.39]$} & 4.396 & {$[0.01,0.02]$} & $<.001$ & 0.087 \\
\hline Average & 0.397 & {$[0.39,0.40]$} & 0.415 & {$[0.41,0.42]$} & 5.257 & {$[0.01,0.03]$} & $<.001$ & 0.104 \\
\hline
\end{tabular}

Panel B: Brightness Difference in Facial Region

\begin{tabular}{|c|c|c|c|c|c|c|c|c|}
\hline & \multicolumn{8}{|c|}{ Women $(\mathrm{N}=10,340)$} \\
\hline & \multicolumn{2}{|c|}{ Heterosexual } & \multicolumn{2}{|c|}{ Lesbian } & \multicolumn{4}{|c|}{ Significance Test } \\
\hline & Mean & $95 \% \mathrm{Cl}$ & Mean & $95 \% \mathrm{Cl}$ & $t$ & $95 \% \mathrm{Cl}$ & $p$ & $d$ \\
\hline Red & 0.552 & {$[0.55,0.55]$} & 0.542 & {$[0.54,0.54]$} & -5.664 & {$[-0.01,-0.01]$} & $<.001$ & 0.077 \\
\hline Green & 0.418 & {$[0.42,0.42]$} & 0.412 & {$[0.41,0.41]$} & -4.041 & {$[-0.01,-0.00]$} & $<.001$ & 0.055 \\
\hline Blue & 0.371 & {$[0.37,0.37]$} & 0.365 & {$[0.36,0.37]$} & -3.453 & {$[-0.01,-0.00]$} & $=.001$ & 0.047 \\
\hline \multirow[t]{4}{*}{ Average } & 0.447 & {$[0.44,0.45]$} & 0.440 & {$[0.44,0.44]$} & -4.722 & {$[-0.01,-0.00]$} & $<.001$ & 0.064 \\
\hline & \multicolumn{8}{|c|}{ Men $(N=5,124)$} \\
\hline & \multicolumn{2}{|c|}{ Heterosexual } & \multicolumn{2}{|r|}{ Gay } & \multicolumn{4}{|c|}{ Significance Test } \\
\hline & Mean & $95 \% \mathrm{Cl}$ & Mean & $95 \% \mathrm{Cl}$ & $t$ & $95 \% \mathrm{Cl}$ & $p$ & $d$ \\
\hline Red & 0.522 & {$[0.52,0.53]$} & 0.549 & {$[0.55,0.55]$} & 10.896 & {$[0.02,0.03]$} & $<.001$ & 0.216 \\
\hline Green & 0.398 & {$[0.40,0.40]$} & 0.414 & {$[0.41,0.42]$} & 7.392 & {$[0.01,0.02]$} & $<.001$ & 0.147 \\
\hline Blue & 0.359 & {$[0.36,0.36]$} & 0.368 & {$[0.37,0.37]$} & 4.393 & {$[0.01,0.01]$} & $<.001$ & 0.087 \\
\hline Average & 0.426 & {$[0.42,0.43]$} & 0.443 & {$[0.44,0.45]$} & 8.252 & {$[0.01,0.02]$} & $<.001$ & 0.164 \\
\hline
\end{tabular}

Panel C: Brightness Difference for Entire Image

\begin{tabular}{|c|c|c|c|c|c|c|c|c|}
\hline & \multicolumn{8}{|c|}{ Women $(\mathrm{N}=10,340)$} \\
\hline & \multicolumn{2}{|c|}{ Heterosexual } & \multicolumn{2}{|c|}{ Lesbian } & \multicolumn{4}{|c|}{ Significance Test } \\
\hline & Mean & $95 \% \mathrm{Cl}$ & Mean & $95 \% \mathrm{Cl}$ & $t$ & $95 \% \mathrm{Cl}$ & $p$ & $d$ \\
\hline Red & 0.951 & {$[0.95,0.96]$} & 0.930 & {$[0.93,0.93]$} & -6.568 & {$[-0.03,-0.02]$} & $<.001$ & 0.089 \\
\hline Green & 0.771 & {$[0.77,0.77]$} & 0.754 & {$[0.75,0.76]$} & -5.638 & {$[-0.02,-0.01]$} & $<.001$ & 0.077 \\
\hline Blue & 0.696 & {$[0.69,0.70]$} & 0.681 & {$[0.68,0.68]$} & -5.325 & {$[-0.02,-0.01]$} & $<.001$ & 0.072 \\
\hline \multirow[t]{4}{*}{ Average } & 0.806 & {$[0.80,0.81]$} & 0.788 & {$[0.78,0.79]$} & -6.198 & {$[-0.02,-0.01]$} & $<.001$ & 0.084 \\
\hline & \multicolumn{8}{|c|}{ Men $(\mathrm{N}=5,124)$} \\
\hline & \multicolumn{2}{|c|}{ Heterosexual } & \multicolumn{2}{|r|}{ Gay } & \multicolumn{4}{|c|}{ Significance Test } \\
\hline & Mean & $95 \% \mathrm{Cl}$ & Mean & $95 \% \mathrm{Cl}$ & $t$ & $95 \% \mathrm{Cl}$ & $p$ & $d$ \\
\hline Red & 0.946 & {$[0.94,0.95]$} & 0.992 & {$[0.99,1.00]$} & 9.701 & {$[0.04,0.06]$} & $<.001$ & 0.192 \\
\hline Green & 0.793 & {$[0.79,0.80]$} & 0.827 & {$[0.82,0.83]$} & 7.645 & {$[0.03,0.04]$} & $<.001$ & 0.152 \\
\hline Blue & 0.731 & {$[0.72,0.74]$} & 0.757 & {$[0.75,0.76]$} & 5.379 & {$[0.02,0.03]$} & $<.001$ & 0.107 \\
\hline Average & 0.823 & {$[0.82,0.83]$} & 0.858 & {$[0.85,0.86]$} & 8.021 & {$[0.03,0.04]$} & $<.001$ & 0.159 \\
\hline
\end{tabular}


Table S8.

Average AUC Results by Different Degrees of Blurring (by Target Width) Compared to AUC of Random (One-pixel Blurred Image of Dataset) for Study $2 b$

\begin{tabular}{|c|c|c|c|c|c|c|c|c|}
\hline \multirow[b]{2}{*}{ Width } & \multicolumn{4}{|c|}{ Women $(\mathrm{N}=10,340)$} & \multicolumn{4}{|c|}{$\operatorname{Men}(\mathrm{N}=5,124)$} \\
\hline & AUC & $95 \% \mathrm{Cl}$ & $p$ & $d$ & AUC & $95 \% \mathrm{Cl}$ & $p$ & $d$ \\
\hline 224 & .702 & {$[.692, .712]$} & $<.001$ & 4.010 & .660 & {$[.645, .675]$} & $<.001$ & 2.610 \\
\hline 112 & .703 & {$[.693, .713]$} & $<.001$ & 4.026 & .661 & {$[.646, .676]$} & $<.001$ & 2.626 \\
\hline 74 & .702 & {$[.692, .712]$} & $<.001$ & 4.011 & .657 & {$[.643, .672]$} & $<.001$ & 2.562 \\
\hline 56 & .703 & {$[.693, .713]$} & $<.001$ & 4.020 & .662 & {$[.647, .677]$} & $<.001$ & 2.641 \\
\hline 44 & .702 & {$[.692, .712]$} & $<.001$ & 4.002 & .668 & {$[.653, .683]$} & $<.001$ & 2.745 \\
\hline 37 & .697 & {$[.687, .707]$} & $<.001$ & 3.889 & .662 & {$[.647, .676]$} & $<.001$ & 2.633 \\
\hline 32 & .691 & {$[.681, .701]$} & $<.001$ & 3.770 & .660 & {$[.645, .674]$} & $<.001$ & 2.596 \\
\hline 28 & .689 & {$[.679, .699]$} & $<.001$ & 3.722 & .657 & {$[.642, .672]$} & $<.001$ & 2.553 \\
\hline 24 & .680 & {$[.670, .691]$} & $<.001$ & 3.535 & .645 & {$[.630, .660]$} & $<.001$ & 2.350 \\
\hline 22 & .670 & {$[.660, .680]$} & $<.001$ & 3.311 & .642 & {$[.627, .658]$} & $<.001$ & 2.302 \\
\hline 20 & .656 & {$[.646, .667]$} & $<.001$ & 3.032 & .636 & {$[.620, .651]$} & $<.001$ & 2.184 \\
\hline 19 & .656 & {$[.646, .667]$} & $<.001$ & 3.022 & .631 & {$[.616, .646]$} & $<.001$ & 2.104 \\
\hline 18 & .646 & {$[.636, .657]$} & $<.001$ & 2.821 & .618 & {$[.603, .633]$} & $<.001$ & 1.888 \\
\hline 17 & .642 & {$[.631, .652]$} & $<.001$ & 2.731 & 615 & {$[.599, .630]$} & $<.001$ & 1.835 \\
\hline 16 & .623 & {$[.612, .634]$} & $<.001$ & 2.356 & .594 & {$[.579, .610]$} & $<.001$ & 1.501 \\
\hline 15 & .618 & {$[.607, .628]$} & $<.001$ & 2.249 & .601 & {$[.585, .616]$} & $<.001$ & 1.607 \\
\hline 14 & .614 & {$[.603, .624]$} & $<.001$ & 2.168 & .585 & {$[.570, .601]$} & $<.001$ & 1.352 \\
\hline 13 & .607 & {$[.596, .618]$} & $<.001$ & 2.044 & .589 & {$[.573, .604]$} & $<.001$ & 1.413 \\
\hline 12 & .599 & {$[.588, .610]$} & $<.001$ & 1.879 & .584 & {$[.569, .600]$} & $<.001$ & 1.337 \\
\hline 11 & .596 & {$[.585, .607]$} & $<.001$ & 1.818 & .581 & {$[.565, .596]$} & $<.001$ & 1.281 \\
\hline 10 & .588 & {$[.577, .599]$} & $<.001$ & 1.666 & .585 & {$[.569, .600]$} & $<.001$ & 1.347 \\
\hline 9 & .590 & {$[.579, .600]$} & $<.001$ & 1.698 & .580 & {$[.565, .596]$} & $<.001$ & 1.272 \\
\hline 8 & .583 & {$[.572, .594]$} & $<.001$ & 1.568 & .586 & {$[.571, .602]$} & $<.001$ & 1.374 \\
\hline 7 & .581 & {$[.570, .592]$} & $<.001$ & 1.530 & .589 & {$[.573, .604]$} & $<.001$ & 1.414 \\
\hline 6 & .572 & {$[.561, .583]$} & $<.001$ & 1.355 & .593 & {$[.578, .609]$} & $<.001$ & 1.484 \\
\hline 5 & .575 & {$[.564, .586]$} & $<.001$ & 1.421 & .578 & {$[.563, .594]$} & $<.001$ & 1.241 \\
\hline 4 & .558 & {$[.547, .569]$} & $<.001$ & 1.090 & .571 & {$[.555, .586]$} & $<.001$ & 1.122 \\
\hline 3 & .559 & {$[.548, .570]$} & $<.001$ & 1.106 & .566 & {$[.550, .581]$} & $<.001$ & 1.038 \\
\hline 2 & .533 & {$[.522, .544]$} & $<.001$ & 0.628 & .568 & {$[.552, .584]$} & $<.001$ & 1.078 \\
\hline 1 & .529 & {$[.518, .540]$} & $<.001$ & 0.546 & .563 & {$[.548, .579]$} & $<.001$ & 1.000 \\
\hline
\end{tabular}

Note. Average AUC was generated by the following steps. First, averaging the predictions across facial images for the same person. Second, comparing the averaged prediction to the observed value to generate the average AUC. 
Table S9.

AUC Results Using One Image by Different Degrees of Blurring (by Target Width) Compared to AUC of Random (One-pixel Blurred Image of Dataset) for Study $2 b$

\begin{tabular}{|c|c|c|c|c|c|c|c|c|}
\hline \multirow[b]{2}{*}{ Width } & \multicolumn{4}{|c|}{ Women $(\mathrm{N}=10,340)$} & \multicolumn{4}{|c|}{$\operatorname{Men}(\mathrm{N}=5,124)$} \\
\hline & AUC & $95 \% \mathrm{Cl}$ & $p$ & $d$ & AUC & $95 \% \mathrm{Cl}$ & $p$ & $d$ \\
\hline 224 & .687 & {$[.677, .697]$} & $<.001$ & 3.681 & .643 & {$[.628, .658]$} & $<.001$ & 2.314 \\
\hline 112 & .688 & {$[.678, .698]$} & $<.001$ & 3.696 & .643 & {$[.628, .658]$} & $<.001$ & 2.315 \\
\hline 74 & .687 & {$[.677, .697]$} & $<.001$ & 3.681 & .642 & {$[.627, .657]$} & $<.001$ & 2.290 \\
\hline 56 & .687 & {$[.677, .698]$} & $<.001$ & 3.691 & .647 & {$[.632, .662]$} & $<.001$ & 2.376 \\
\hline 44 & .686 & {$[.676, .696]$} & $<.001$ & 3.652 & .646 & {$[.631, .661]$} & $<.001$ & 2.369 \\
\hline 37 & .680 & {$[.670, .690]$} & $<.001$ & 3.528 & .640 & {$[.625, .655]$} & $<.001$ & 2.261 \\
\hline 32 & .673 & {$[.663, .683]$} & $<.001$ & 3.380 & .638 & {$[.623, .653]$} & $<.001$ & 2.228 \\
\hline 28 & .670 & {$[.660, .681]$} & $<.001$ & 3.325 & 638 & {$[.623, .653]$} & $<.001$ & 2.224 \\
\hline 24 & .660 & {$[.650, .670]$} & $<.001$ & 3.109 & .623 & {$[.608, .638]$} & $<.001$ & 1.976 \\
\hline 22 & .650 & {$[.640, .661]$} & $<.001$ & 2.904 & .619 & {$[.603, .634]$} & $<.001$ & 1.901 \\
\hline 20 & .637 & {$[.627, .648]$} & $<.001$ & 2.643 & .617 & {$[.602, .633]$} & $<.001$ & 1.881 \\
\hline 19 & .634 & {$[.623, .644]$} & $<.001$ & 2.569 & .610 & {$[.595, .625]$} & $<.001$ & 1.757 \\
\hline 18 & .630 & {$[.619, .641]$} & $<.001$ & 2.490 & 601 & {$[.586, .616]$} & $<.001$ & 1.613 \\
\hline 17 & .620 & {$[.609, .631]$} & $<.001$ & 2.298 & .598 & {$[.582, .613]$} & $<.001$ & 1.559 \\
\hline 16 & .606 & {$[.595, .617]$} & $<.001$ & 2.023 & .581 & {$[.566, .597]$} & $<.001$ & 1.289 \\
\hline 15 & .598 & {$[.587, .609]$} & $<.001$ & 1.869 & .586 & {$[.570, .602]$} & $<.001$ & 1.367 \\
\hline 14 & .594 & {$[.583, .605]$} & $<.001$ & 1.789 & .571 & {$[.555, .586]$} & $<.001$ & 1.121 \\
\hline 13 & .594 & {$[.583, .605]$} & $<.001$ & 1.779 & .575 & {$[.559, .590]$} & $<.001$ & 1.182 \\
\hline 12 & .585 & {$[.574, .596]$} & $<.001$ & 1.610 & .573 & {$[.558, .589]$} & $<.001$ & 1.159 \\
\hline 11 & .582 & {$[.571, .593]$} & $<.001$ & 1.556 & .570 & {$[.554, .585]$} & $<.001$ & 1.104 \\
\hline 10 & .575 & {$[.564, .585]$} & $<.001$ & 1.408 & .571 & {$[.555, .586]$} & $<.001$ & 1.123 \\
\hline 9 & .576 & {$[.565, .587]$} & $<.001$ & 1.441 & .566 & {$[.550, .581]$} & $<.001$ & 1.041 \\
\hline 8 & .569 & {$[.558, .580]$} & $<.001$ & 1.300 & .570 & {$[.554, .585]$} & $<.001$ & 1.105 \\
\hline 7 & .567 & {$[.556, .578]$} & $<.001$ & 1.272 & .574 & {$[.559, .590]$} & $<.001$ & 1.177 \\
\hline 6 & .562 & {$[.551, .573]$} & $<.001$ & 1.177 & .581 & {$[.566, .597]$} & $<.001$ & 1.291 \\
\hline 5 & .565 & {$[.554, .576]$} & $<.001$ & 1.228 & .564 & {$[.549, .580]$} & $<.001$ & 1.021 \\
\hline 4 & .550 & {$[.539, .561]$} & $<.001$ & 0.948 & .559 & {$[.544, .575]$} & $<.001$ & 0.936 \\
\hline 3 & .546 & {$[.535, .557]$} & $<.001$ & 0.872 & .552 & {$[.536, .568]$} & $<.001$ & 0.819 \\
\hline 2 & .527 & {$[.516, .538]$} & $<.001$ & 0.509 & .548 & {$[.532, .564]$} & $<.001$ & 0.760 \\
\hline 1 & .523 & {$[.512, .534]$} & $<.001$ & 0.437 & .548 & {$[.532, .564]$} & $<.001$ & 0.758 \\
\hline
\end{tabular}


Table S10.

Average Accuracy Results by Different Degrees of Blurring (by Target Width) for Study $2 b$

\begin{tabular}{|c|c|c|c|c|c|c|c|c|}
\hline \multirow[b]{2}{*}{ Width } & \multicolumn{4}{|c|}{ Women } & \multicolumn{4}{|c|}{ Men } \\
\hline & Accuracy & Precision & Recall & F1 & Accuracy & Precision & Recall & F1 \\
\hline 224 & $64.76 \%$ & $64.63 \%$ & $65.20 \%$ & $64.91 \%$ & $61.32 \%$ & $61.58 \%$ & $60.19 \%$ & $60.88 \%$ \\
\hline 112 & $64.93 \%$ & $64.79 \%$ & $65.40 \%$ & $65.09 \%$ & $61.65 \%$ & $61.94 \%$ & $60.46 \%$ & $61.19 \%$ \\
\hline 74 & $64.89 \%$ & $64.71 \%$ & $65.53 \%$ & $65.12 \%$ & $61.28 \%$ & $61.50 \%$ & $60.30 \%$ & $60.90 \%$ \\
\hline 56 & $64.92 \%$ & $64.69 \%$ & $65.73 \%$ & $65.20 \%$ & $61.44 \%$ & $61.59 \%$ & $60.77 \%$ & $61.18 \%$ \\
\hline 44 & $64.76 \%$ & $64.59 \%$ & $65.34 \%$ & $64.96 \%$ & $62.24 \%$ & $62.46 \%$ & $61.36 \%$ & $61.90 \%$ \\
\hline 37 & $64.11 \%$ & $63.76 \%$ & $65.40 \%$ & $64.57 \%$ & $61.59 \%$ & $61.56 \%$ & $61.75 \%$ & $61.65 \%$ \\
\hline 32 & $64.52 \%$ & $64.16 \%$ & $65.76 \%$ & $64.95 \%$ & $62.06 \%$ & $62.37 \%$ & $60.81 \%$ & $61.58 \%$ \\
\hline 28 & $63.97 \%$ & $63.58 \%$ & $65.44 \%$ & $64.49 \%$ & $61.44 \%$ & $61.84 \%$ & $59.72 \%$ & $60.76 \%$ \\
\hline 24 & $63.42 \%$ & $63.04 \%$ & $64.89 \%$ & $63.95 \%$ & $60.40 \%$ & $60.85 \%$ & $58.35 \%$ & $59.57 \%$ \\
\hline 22 & $62.66 \%$ & $62.23 \%$ & $64.43 \%$ & $63.31 \%$ & $60.85 \%$ & $61.53 \%$ & $57.92 \%$ & $59.67 \%$ \\
\hline 20 & $61.42 \%$ & $61.01 \%$ & $63.31 \%$ & $62.14 \%$ & $60.44 \%$ & $61.19 \%$ & $57.10 \%$ & $59.08 \%$ \\
\hline 19 & $62.26 \%$ & $61.60 \%$ & $65.11 \%$ & $63.31 \%$ & $59.89 \%$ & $60.57 \%$ & $56.71 \%$ & $58.58 \%$ \\
\hline 18 & $61.33 \%$ & $60.75 \%$ & $64.06 \%$ & $62.36 \%$ & $58.24 \%$ & $58.54 \%$ & $56.44 \%$ & $57.47 \%$ \\
\hline 17 & $60.38 \%$ & $59.86 \%$ & $63.02 \%$ & $61.40 \%$ & $58.70 \%$ & $59.18 \%$ & $56.13 \%$ & $57.61 \%$ \\
\hline 16 & $59.31 \%$ & $58.56 \%$ & $63.69 \%$ & $61.02 \%$ & $56.79 \%$ & $56.79 \%$ & $56.83 \%$ & $56.81 \%$ \\
\hline 15 & $58.17 \%$ & $57.71 \%$ & $61.18 \%$ & $59.39 \%$ & $56.91 \%$ & $57.21 \%$ & $54.84 \%$ & $56.00 \%$ \\
\hline 14 & $58.14 \%$ & $57.68 \%$ & $61.16 \%$ & $59.37 \%$ & $56.64 \%$ & $56.69 \%$ & $56.25 \%$ & $56.47 \%$ \\
\hline 13 & $57.55 \%$ & $57.17 \%$ & $60.19 \%$ & $58.65 \%$ & $56.44 \%$ & $56.47 \%$ & $56.21 \%$ & $56.34 \%$ \\
\hline 12 & $57.43 \%$ & $57.09 \%$ & $59.79 \%$ & $58.41 \%$ & $56.03 \%$ & $55.92 \%$ & $56.99 \%$ & $56.45 \%$ \\
\hline 11 & $57.01 \%$ & $56.77 \%$ & $58.82 \%$ & $57.78 \%$ & $56.28 \%$ & $56.20 \%$ & $56.95 \%$ & $56.57 \%$ \\
\hline 10 & $56.61 \%$ & $56.33 \%$ & $58.76 \%$ & $57.52 \%$ & $56.67 \%$ & $56.42 \%$ & $58.63 \%$ & $57.50 \%$ \\
\hline 9 & $56.23 \%$ & $55.98 \%$ & $58.28 \%$ & $57.11 \%$ & $56.19 \%$ & $56.05 \%$ & $57.30 \%$ & $56.67 \%$ \\
\hline 8 & $56.32 \%$ & $56.22 \%$ & $57.08 \%$ & $56.65 \%$ & $56.23 \%$ & $56.12 \%$ & $57.10 \%$ & $56.61 \%$ \\
\hline 7 & $55.84 \%$ & $55.69 \%$ & $57.16 \%$ & $56.41 \%$ & $56.83 \%$ & $56.81 \%$ & $56.99 \%$ & $56.90 \%$ \\
\hline 6 & $55.05 \%$ & $54.91 \%$ & $56.50 \%$ & $55.69 \%$ & $56.93 \%$ & $56.85 \%$ & $57.53 \%$ & $57.19 \%$ \\
\hline 5 & $55.55 \%$ & $55.58 \%$ & $55.32 \%$ & $55.45 \%$ & $55.89 \%$ & $55.73 \%$ & $57.30 \%$ & $56.51 \%$ \\
\hline 4 & $54.04 \%$ & $54.02 \%$ & $54.29 \%$ & $54.16 \%$ & $54.98 \%$ & $54.93 \%$ & $55.46 \%$ & $55.20 \%$ \\
\hline 3 & $54.35 \%$ & $54.15 \%$ & $56.77 \%$ & $55.43 \%$ & $54.88 \%$ & $54.86 \%$ & $55.07 \%$ & $54.97 \%$ \\
\hline 2 & $52.79 \%$ & $52.76 \%$ & $53.29 \%$ & $53.02 \%$ & $55.13 \%$ & $55.10 \%$ & $55.46 \%$ & $55.28 \%$ \\
\hline 1 & $52.34 \%$ & $52.36 \%$ & $51.99 \%$ & $52.17 \%$ & $54.18 \%$ & $54.16 \%$ & $54.33 \%$ & $54.25 \%$ \\
\hline Random & $50.00 \%$ & $50.00 \%$ & $100.00 \%$ & $66.67 \%$ & $50.00 \%$ & $50.00 \%$ & $100.00 \%$ & $66.67 \%$ \\
\hline
\end{tabular}

Note. Average accuracy was generated by the following steps. First, averaging the predictions across facial images for the same person. Second, comparing the averaged prediction to the observed value to generate the average accuracy. 
Table S11.

Accuracy Results for One Image Only by Different Degrees of Blurring (by Target Width) for Study $2 b$

\begin{tabular}{|c|c|c|c|c|c|c|c|c|}
\hline \multirow[b]{2}{*}{ Width } & \multicolumn{4}{|c|}{ Women } & \multicolumn{4}{|c|}{ Men } \\
\hline & Accuracy & Precision & Recall & F1 & Accuracy & Precision & Recall & F1 \\
\hline 224 & $63.83 \%$ & $63.59 \%$ & $64.70 \%$ & $64.14 \%$ & $59.70 \%$ & $59.89 \%$ & $58.74 \%$ & $59.31 \%$ \\
\hline 112 & $63.38 \%$ & $63.10 \%$ & $64.41 \%$ & $63.75 \%$ & $60.19 \%$ & $60.47 \%$ & $58.82 \%$ & $59.64 \%$ \\
\hline 74 & $63.63 \%$ & $63.29 \%$ & $64.87 \%$ & $64.07 \%$ & $60.25 \%$ & $60.50 \%$ & $59.02 \%$ & $59.75 \%$ \\
\hline 56 & $63.40 \%$ & $63.09 \%$ & $64.60 \%$ & $63.84 \%$ & $60.62 \%$ & $60.85 \%$ & $59.56 \%$ & $60.20 \%$ \\
\hline 44 & $63.41 \%$ & $63.09 \%$ & $64.64 \%$ & $63.86 \%$ & $60.34 \%$ & $60.56 \%$ & $59.33 \%$ & $59.94 \%$ \\
\hline 37 & $63.00 \%$ & $62.53 \%$ & $64.85 \%$ & $63.67 \%$ & $59.78 \%$ & $59.91 \%$ & $59.13 \%$ & $59.52 \%$ \\
\hline 32 & $62.75 \%$ & $62.38 \%$ & $64.24 \%$ & $63.29 \%$ & $60.34 \%$ & $60.77 \%$ & $58.35 \%$ & $59.54 \%$ \\
\hline 28 & $62.36 \%$ & $61.91 \%$ & $64.24 \%$ & $63.05 \%$ & $59.62 \%$ & $59.99 \%$ & $57.77 \%$ & $58.86 \%$ \\
\hline 24 & $61.76 \%$ & $61.28 \%$ & $63.89 \%$ & $62.56 \%$ & $58.43 \%$ & $58.83 \%$ & $56.17 \%$ & $57.47 \%$ \\
\hline 22 & $60.84 \%$ & $60.22 \%$ & $63.87 \%$ & $61.99 \%$ & $58.47 \%$ & $59.12 \%$ & $54.92 \%$ & $56.94 \%$ \\
\hline 20 & $59.36 \%$ & $58.94 \%$ & $61.72 \%$ & $60.30 \%$ & $58.10 \%$ & $58.76 \%$ & $54.33 \%$ & $56.46 \%$ \\
\hline 19 & $59.95 \%$ & $59.37 \%$ & $63.08 \%$ & $61.16 \%$ & $57.75 \%$ & $58.21 \%$ & $54.92 \%$ & $56.52 \%$ \\
\hline 18 & $59.84 \%$ & $59.27 \%$ & $62.86 \%$ & $61.02 \%$ & $56.71 \%$ & $56.94 \%$ & $55.07 \%$ & $55.99 \%$ \\
\hline 17 & $58.32 \%$ & $57.90 \%$ & $60.97 \%$ & $59.39 \%$ & $56.83 \%$ & $57.05 \%$ & $55.27 \%$ & $56.15 \%$ \\
\hline 16 & $57.91 \%$ & $57.32 \%$ & $61.97 \%$ & $59.55 \%$ & $55.99 \%$ & $56.06 \%$ & $55.46 \%$ & $55.76 \%$ \\
\hline 15 & $56.91 \%$ & $56.54 \%$ & $59.73 \%$ & $58.09 \%$ & $55.89 \%$ & $56.13 \%$ & $53.98 \%$ & $55.03 \%$ \\
\hline 14 & $56.72 \%$ & $56.35 \%$ & $59.63 \%$ & $57.95 \%$ & $55.21 \%$ & $55.22 \%$ & $55.15 \%$ & $55.18 \%$ \\
\hline 13 & $56.46 \%$ & $56.14 \%$ & $59.03 \%$ & $57.55 \%$ & $55.00 \%$ & $54.99 \%$ & $55.04 \%$ & $55.01 \%$ \\
\hline 12 & $56.25 \%$ & $56.02 \%$ & $58.14 \%$ & $57.06 \%$ & $55.09 \%$ & $54.97 \%$ & $56.36 \%$ & $55.66 \%$ \\
\hline 11 & $55.93 \%$ & $55.75 \%$ & $57.52 \%$ & $56.62 \%$ & $55.37 \%$ & $55.23 \%$ & $56.64 \%$ & $55.93 \%$ \\
\hline 10 & $55.34 \%$ & $55.17 \%$ & $57.00 \%$ & $56.07 \%$ & $55.27 \%$ & $55.05 \%$ & $57.42 \%$ & $56.21 \%$ \\
\hline 9 & $55.08 \%$ & $54.90 \%$ & $56.87 \%$ & $55.87 \%$ & $55.13 \%$ & $54.92 \%$ & $57.30 \%$ & $56.08 \%$ \\
\hline 8 & $55.28 \%$ & $55.16 \%$ & $56.48 \%$ & $55.81 \%$ & $54.78 \%$ & $54.68 \%$ & $55.89 \%$ & $55.28 \%$ \\
\hline 7 & $54.85 \%$ & $54.80 \%$ & $55.44 \%$ & $55.12 \%$ & $55.52 \%$ & $55.37 \%$ & $56.99 \%$ & $56.16 \%$ \\
\hline 6 & $54.36 \%$ & $54.24 \%$ & $55.82 \%$ & $55.02 \%$ & $56.67 \%$ & $56.58 \%$ & $57.38 \%$ & $56.98 \%$ \\
\hline 5 & $54.65 \%$ & $54.69 \%$ & $54.27 \%$ & $54.48 \%$ & $55.31 \%$ & $55.16 \%$ & $56.71 \%$ & $55.93 \%$ \\
\hline 4 & $53.33 \%$ & $53.33 \%$ & $53.25 \%$ & $53.29 \%$ & $54.84 \%$ & $54.77 \%$ & $55.54 \%$ & $55.16 \%$ \\
\hline 3 & $53.17 \%$ & $53.02 \%$ & $55.69 \%$ & $54.32 \%$ & $53.98 \%$ & $53.96 \%$ & $54.29 \%$ & $54.12 \%$ \\
\hline 2 & $51.81 \%$ & $51.78 \%$ & $52.65 \%$ & $52.21 \%$ & $53.01 \%$ & $52.98 \%$ & $53.43 \%$ & $53.21 \%$ \\
\hline 1 & $51.94 \%$ & $51.96 \%$ & $51.47 \%$ & $51.72 \%$ & $53.10 \%$ & $53.11 \%$ & $53.01 \%$ & $53.06 \%$ \\
\hline Random & $50.00 \%$ & $50.00 \%$ & $100.00 \%$ & $66.67 \%$ & $50.00 \%$ & $50.00 \%$ & $100.00 \%$ & $66.67 \%$ \\
\hline
\end{tabular}


Figure S1.

An Example Rendering of Image Augmentations Applied in Studies $1 c$ and $2 b$

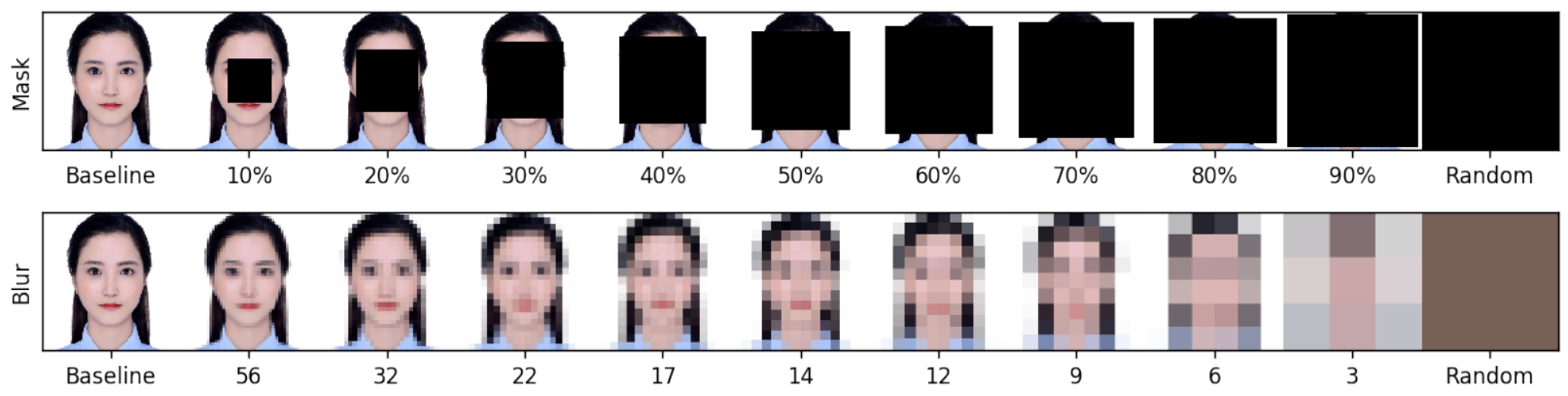


Figure S2.

A Plot of the Target Width Used to Downsize Images for Study $2 b$

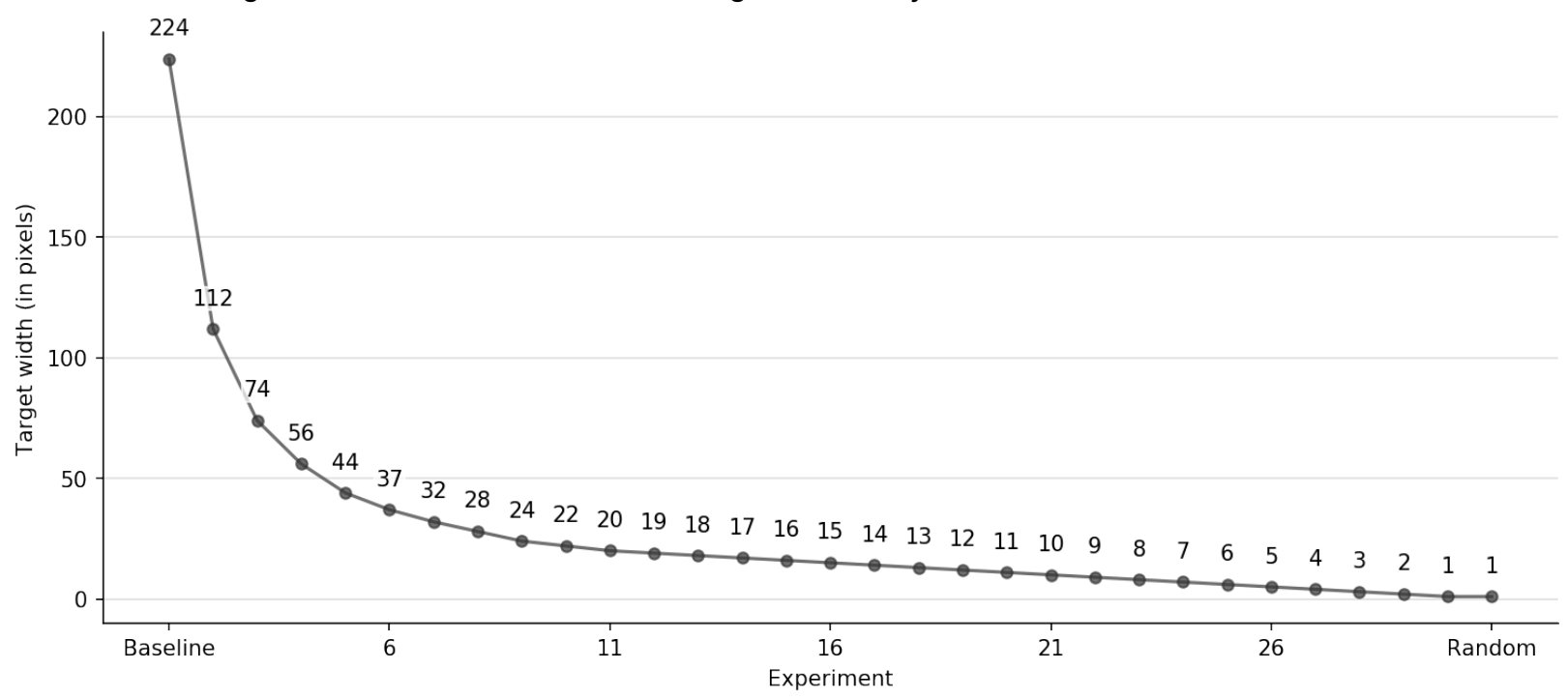

